\title{
Universal thermodynamics in the Kitaev fractional liquid
}

\author{
Han Li $\odot,{ }^{1}$ Dai-Wei Qu, ${ }^{1}$ Hao-Kai Zhang, ${ }^{1}$ Yi-Zhen Jia, ${ }^{1}$ Shou-Shu Gong, ${ }^{1, *}$ Yang Qi, $, 2,3,4, \dagger$ and Wei Li ${ }^{1,5, *}$ \\ ${ }^{1}$ Key Laboratory of Micro-Nano Measurement-Manipulation and Physics (Ministry of Education), \\ School of Physics, Beihang University, Beijing 100191, China \\ ${ }^{2}$ State Key Laboratory of Surface Physics, Fudan University, Shanghai 200433, China \\ ${ }^{3}$ Center for Field Theory and Particle Physics, Department of Physics, Fudan University, Shanghai 200433, China \\ ${ }^{4}$ Collaborative Innovation Center of Advanced Microstructures, Nanjing 210093, China \\ ${ }^{5}$ International Research Institute of Multidisciplinary Science, Beihang University, Beijing 100191, China
}

(Received 8 June 2020; revised 8 September 2020; accepted 10 September 2020; published 2 October 2020)

\begin{abstract}
In the Kitaev honeycomb model, the quantum spin fractionalizes into itinerant Majorana fermion and gauge flux upon cooling, leading to rich experimental ramifications at finite temperature and an upsurge of research interest. However, accurate modelings of the Kitaev materials by including realistic couplings beyond the pure Kitaev model constitute a major challenge for the community. With recently developed exponential tensor-network approach, we clear the pathway and perform finite-temperature simulations of the extended Kitaev model with additional interactions common in real materials. At intermediate temperature, we find an emergent Curie law of magnetic susceptibility and a stripy spin-structure factor characterizing the robust Kitaev fractional liquid. With this insight, we revisit the susceptibility measurements of $\mathrm{Na}_{2} \mathrm{IrO}_{3}$ and $\alpha-\mathrm{RuCl}_{3}$ and find evidence of ferromagnetic Kitaev coupling and finite-temperature fractionalization. Bridging the gap between theories and experiments with the state-of-the-art tensor-network simulations, our findings provide guidance for future experimental exploration of the spin liquids in Kitaev materials by thermodynamic measurements and spin-resolved structure factor probes.
\end{abstract}

DOI: 10.1103/PhysRevResearch.2.043015

\section{INTRODUCTION}

Quantum spin liquids realize a novel class of quantum states of matter [1-3] which represent the nonmagnetic states with emergent long-range entanglement and fractionalized excitations in frustrated magnets [4,5]. After extensive research for decades, the exactly soluble Kitaev model of the honeycomb lattice [6], which has three types of bond-directional Ising couplings in the trivalent lattice, was found as a prototypical system that can realize both gapped and gapless spin liquid states with spins fractionalized into itinerant and localized Majorana fermions [6,7]. In particular, in the gapless spin liquid phase, time-reversal symmetry-breaking perturbations such as a magnetic field can immediately induce a gapped non-Abelian chiral spin liquid, which has potential application for topological quantum computation [8].

The pursuit of the materialization of the intriguing Kitaev spin liquid (KSL) states has triggered a competition in the search for crystalline Kitaev-like materials [9-11]. Fortunately, there are Mott insulators with strong spin-orbit

\footnotetext{
*shoushu.gong@buaa.edu.cn

†qiyang@fudan.edu.cn

${ }^{\ddagger}$ w.li@buaa.edu.cn
}

Published by the American Physical Society under the terms of the Creative Commons Attribution 4.0 International license. Further distribution of this work must maintain attribution to the author(s) and the published article's title, journal citation, and DOI. coupling and an effective pseudospin $\frac{1}{2}$ on the honeycomb lattice that can realize the highly anisotropic couplings. These materials have been intensively studied in recent years, including the iridate family, such as $A_{2} \mathrm{IrO}_{3}(A=\mathrm{Li}$ and $\mathrm{Na})$ [12-18] and $\mathrm{H}_{3} \mathrm{LiIr}_{2} \mathrm{O}_{6}$ [19], and the ruthenate family, such as $\alpha-\mathrm{RuCl}_{3}$ [20-24]. Nonetheless, due to the couplings beyond the Kitaev model such as the Heisenberg, off-diagonal $\Gamma$, and further-neighbor interactions in real materials, semiclassical magnetic orders appear at low temperature $T$ in most of these KSL candidate compounds. Surprisingly, signatures of fractionalization in quantum spins can nevertheless be detected at intermediate temperature in some Kitaev materials [17,2224], even when the low- $T$ states are classically ordered. These exciting observations and the spin liquid phase found in the presence of magnetic fields strongly suggest that these materials should be in proximity to the KSL phase and imminently call for systematic understanding of the thermodynamic properties of these Kitaev materials.

For the pure Kitaev model, the finite- $T$ fractionalization of spins has been well established via the Majorana-based quantum Monte Carlo (QMC) simulations [25-27], which found two temperature scales and an intermediate regime with unconventional properties [25-29]. This provides an understanding of the exotic equilibrium [24] and transport measurements [30] in the Kitaev materials. Nonetheless, the gap between experiments and theoretical understanding is still apparent. Since the non-Kitaev interactions and magnetic field $[31,32]$ spoil the exact solution and restrict QMC simulations to a relatively high temperature [33], little is known about the fractionalization of spins in the extended Kitaev models. 
In this work we employ the exponential tensor renormalization group (XTRG) [34-37] at finite temperature to explore the extended Kitaev model with additional Heisenberg and $\Gamma$ interactions, which denies QMC simulations. We perform accurate thermodynamic calculations down to very low temperature and find an emergent Curie law of magnetic susceptibility below a high- $T$ scale $T_{H}$ (and above the low- $T$ scale $T_{L}$ ), due to the fluctuating gauge fluxes and the predominant bond-directional nearest-neighbor $(\mathrm{NN})$ spin correlations. In addition, the well-recognized characteristic features at intermediate temperature, including the fractional thermal-entropy plateau and the linear- $T$ specific-heat scaling, also clearly persist in the extended Kitaev model.

Through exploring these robust finite- $T$ features, we find a generic scenario in the extended Kitaev models relating to the realistic Kitaev materials: A fractionalization of the quantum spin takes place as temperature decreases, and half of the spin degrees of freedom, i.e., the itinerant Majorana fermions, release a large part of the entropy below $T_{H}$, accompanied by a saturated short-range spin correlation; meanwhile, the other half, the gauge fluxes, fluctuate strongly until near the much lower temperature $T_{L}$. In between the two temperature scales resides a unique finite- $T$ quantum state dubbed the Kitaev fractional liquid, which constitutes a precursor of the quantum spin liquids.

Based on our XTRG simulations, we reveal that the fractional liquid regime does exist at intermediate temperature, for an extended range of external fields or non-Kitaev terms including the Heisenberg or $\Gamma$ couplings, even when the KSL ground state is altered. These results therefore shed light on the exotic finite- $T$ quantum states and intriguing properties of the Kitaev materials. In particular, with insight from the universal susceptibility scaling, we revisit the magnetic susceptibility data of $\mathrm{Na}_{2} \mathrm{IrO}_{3}$ and $\alpha-\mathrm{RuCl}_{3}$ and find an evident signature of Curie susceptibility scaling in the two prominent Kitaev materials, suggesting the existence of the fractional liquid therein. We also propose the spin-correlation diagnostics of the fractional quantum liquid, which is available through spin-resolved neutron or x-ray diffusion scatterings.

The rest of the paper is organized as follows. In Sec. II we introduce the extended Kitaev model and the XTRG method for finite- $T$ simulations. Sections III-V present our main results on the universal thermodynamic properties and spin-correlation characterization of the intermediate- $T$ Kitaev fractional liquid that is robust against additional interactions. In Sec. VI we discuss the relevance of universal susceptibility scaling in two Kitaev materials, $\mathrm{Na}_{2} \mathrm{IrO}_{3}$ and $\alpha-\mathrm{RuCl}_{3}$, and propose a spin-structure diagnosis of the fractional liquid. In Sec. VII we summarize our main conclusions and discuss their implications in future studies of the Kitaev model and materials.

\section{MODEL AND METHODS}

We study the celebrated Kitaev honeycomb model with various couplings

$H=\sum_{\langle i, j\rangle_{\gamma}}\left[K_{\gamma} S_{i}^{\gamma} S_{j}^{\gamma}+J \mathbf{S}_{i} \cdot \mathbf{S}_{j}+\Gamma\left(S_{i}^{\alpha} S_{j}^{\beta}+S_{i}^{\beta} S_{j}^{\alpha}\right)\right]+\sum_{i} h S_{i}^{z}$,

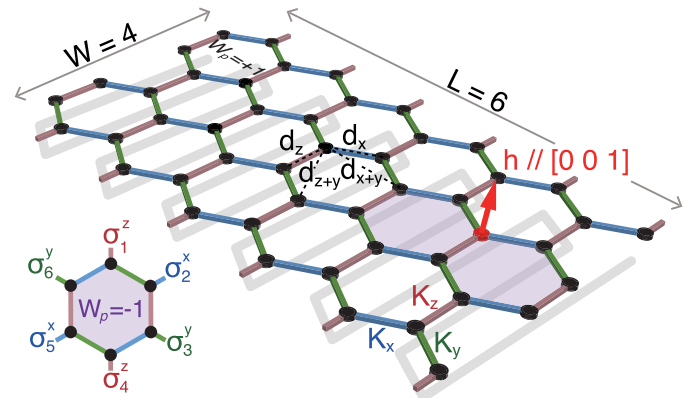

FIG. 1. Cylindrical honeycomb lattice of size YC $4 \times 6 \times 2$ illustrated with the snakelike quasi-1D mapping path (underlying gray lines), adopted in the XTRG calculations. Three types of Kitaev bonds $K_{x}, K_{y}$, and $K_{z}$ are marked in blue, green, and red, respectively. The $\mathbb{Z}_{2}$ flux $W_{P}=\sigma_{1}^{z} \sigma_{2}^{x} \sigma_{3}^{y} \sigma_{4}^{z} \sigma_{5}^{x} \sigma_{6}^{y}=+1$ (hexagon in white) or -1 (purple), with $\sigma_{i}^{\gamma}=2 S_{i}^{\gamma}$, can be pair flipped by applying a magnetic field $h \|$ [001] on the spin-space cubic axis. Typical nearest and next-nearest bonds $d_{x}, d_{z}, d_{x+y}$, and $d_{x+z}$ are indicated by the dashed lines.

where $\mathbf{S}_{i}=\left\{S_{i}^{x}, S_{i}^{y}, S_{i}^{z}\right\}$ represent the spin- $\frac{1}{2}$ vector operator at site $i$. The three cubic coordinates $\{\alpha, \beta, \gamma\}$ represent $\{x, y, z\}$ up to a cyclic permutation and $\langle i, j\rangle_{\gamma}$ denotes the $\mathrm{NN} \gamma$ bond (see Fig. 1) with an Ising coupling $K_{\gamma}$. The non-Kitaev terms include the NN isotropic Heisenberg coupling $J$ and an offdiagonal $\Gamma$ interaction. Moreover, we apply an external field $h$ coupled to the $S^{z}$ component, which is along the [001] spinspace direction and tilts an angle of $45^{\circ}$ from the honeycomb $a b$ plane (see Fig. 1) in typical Kitaev materials.

For the pure Kitaev model, spin can be represented as localized $\left(b_{i}^{\gamma}\right)$ and itinerant Majorana fermions $\left(c_{i}\right)$ through the parton construction $S_{i}^{\gamma}=\frac{i}{2} b_{i}^{\gamma} c_{i}$ [8]. The itinerant Majorana fermions can be related to the building up of spin correlation $\left\langle S_{i}^{z} S_{j}^{z}\right\rangle=u_{i j}^{z}\left\langle c_{i} c_{j}\right\rangle$, and $u_{i j}^{\gamma}=b_{i}^{\gamma} b_{j}^{\gamma}= \pm i$ represents a $\mathbb{Z}_{2}$ gauge connection. The product of $u_{i j}^{\gamma}$ on the six edges of a hexagon gives rise to the flux $W_{P}= \pm 1$ as illustrated in Fig. 1, which is conserved in the pure Kitaev case.

For the typical Kitaev materials $\mathrm{Na}_{2} \mathrm{IrO}_{3}$ and $\alpha-\mathrm{RuCl}_{3}$, the predominant Kitaev interactions have been widely accepted to be ferromagnetic (FM) $[10,24,33,38-40]$. Thus, we choose $K_{\gamma}<0$ henceforth and set $K_{z}=-1$ as the fixed energy scale. The $\Gamma$ term is suggested to be of next-to-leading order and with $\Gamma>0$ in $\alpha-\mathrm{RuCl}_{3}[41,42]$. On the other hand, in $\mathrm{Na}_{2} \mathrm{IrO}_{3}$ the Heisenberg coupling is believed to play an important role and is proposed to be antiferromagnetic (AF) $[38,43]$. Thus, in our simulations below we introduce an additional AF Heisenberg or $\Gamma$ term with $J, \Gamma>0$ and focus on the finite- $T$ quantum states of the extended Kitaev model.

We use the XTRG method $[34,36]$ to study the extended Kitaev model in this work. To start the procedure, we exploit a quasi-one-dimensional (quasi-1D) mapping and express the Hamiltonian of the lattice model as a compact matrix product operator (MPO). As shown in Fig. 1, the cylinder is mapped, via a snakelike path, into a quasi-1D system illustrated by the gray lines in the shadow. Given the MPO representation of the Hamiltonian $H$, we can represent the high-temperature density matrix also as an MPO via the series expansion (see details in Appendix A). After that, in the course of the 
exponential cooling procedure, we keep squaring the density matrices to reach various lower temperatures. The free energy as well as other thermodynamic quantities can be computed at each temperature point via the thermofield double formalism, through a bilayer tensor trace [44].

We work on the cylindrical lattice $\mathrm{YC} W \times L \times 2$ (see Fig. 1), with width $W$ (up to 4) and length $L$ (up to 6), thus total site $N=2 W L$, which reproduces results in excellent agreement with benchmark exact diagonalization (ED) on small system sizes as well as large-scale Majorana QMC simulations (see Appendix A). From comprehensive benchmark results, we see that finite-size effects are in most cases modest, at least for the intermediate- $T$ regime of main focus in the present study. This is in contrast with the ground-state calculations and can be ascribed to the extremely-short-range correlations at intermediate temperature, even under nonKitaev interactions (see Appendix B).

Moreover, as there are two characteristic temperature scales in the Kitaev model that are typically separated by more than one order of magnitude, our XTRG approach that cools down the system exponentially in the temperature scale turns out to be very efficient to address this problem. Therefore, we can exploit the XTRG method that is free of the sign problem to simulate the extended Kitaev model down to extremely low temperature $T \simeq 0.005$. In practical calculations we retain bond dimension up to $D=600$ that is capable of describing the thermal quantum states accurately and produces well-converged thermodynamic results (Appendix C). We have also employed the density matrix renormalization group (DMRG) method to simulate the ground state, confirming the XTRG results at very low temperature.

\section{KITAEV FRACTIONAL LIQUID}

\section{A. Pure ferromagnetic Kitaev model at finite temperature}

It has been well established that the itinerant and localized Majorana fermions can give rise to the two temperature scales $T_{H}$ and $T_{L}$, respectively. At intermediate temperature $T_{L} \lesssim T \lesssim$ $T_{H}$, an exotic fractional quantum liquid emerges. To start with, we simulate finite- $T$ properties of the isotropic FM Kitaev model $\left(K_{x, y, z}=-1\right)$ by XTRG on a YC $4 \times 6 \times 2$ cylinder. In Fig. 2(a) we plot the spin correlation $\left\langle S_{i}^{z} S_{j}^{z}\right\rangle$ measured along the NN bonds $d_{z}$ and $d_{x}\left(d_{y}\right.$ always leads to the same results as $d_{x}$ and thus is not shown) and next-nearest ones $d_{z+y}$ and $d_{x+y}$ (as depicted in Fig. 1). From the zero-field XTRG results, we find that only the nearest- $d_{z}$-bond correlations is established below $T_{H}$ and the rest remain small down to the lowest temperature. Thus the spin correlations $\left\langle S_{i}^{\gamma} S_{j}^{\gamma}\right\rangle$ are nonzero only on the NN $\gamma$ bond, i.e., they are extremely short range and bond oriented, in full agreement with the analytical results $[45,46]$.

In fact, the itinerant Majorana fermions release most of their entropy and the NN spin correlation is established at around $T_{H}$ [Fig. 2(a)]. On the other hand, the rest half of the spins, $\mathbb{Z}_{2}$ fluxes, strongly fluctuate until the low- $T$ scale $T_{L}$, as evidenced by small $W_{P}$ above $T_{L}$ in Fig. 2(c). Correspondingly, in Fig. 2(d) the thermal entropy exhibits a quasiplateau with $S \simeq \frac{1}{2} \ln 2$, which constitutes a thermodynamic signature of the intermediate- $T$ Kitaev fractional liquid. (a)

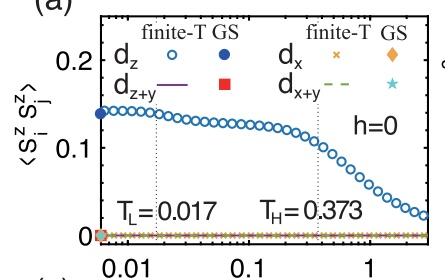

(b)

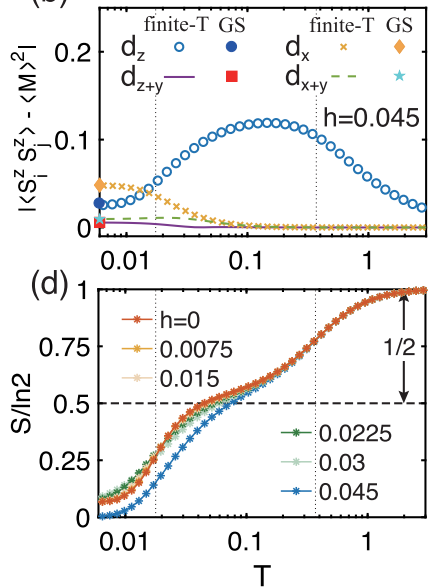

FIG. 2. Spin correlations $\left\langle S_{i}^{z} S_{j}^{z}\right\rangle$ with (a) $h=0$ and (b) $h=0.045$ on bond $\langle i, j\rangle=d_{x}, d_{z}, d_{x+y}$, and $d_{x+z}$ as depicted in Fig. 1, where the $\mathrm{NN}$ spin correlations are established at $T_{H} \simeq 0.373$ and converge to the ground-state (GS) DMRG results (closed symbols) at low temperature. In (b) the mean magnetization squared $\langle M\rangle^{2}$ has been subtracted in the correlation data (see the text). (c) Thermal average of flux $W_{P}$ and (d) thermal entropy $S$ results under various fields. As temperature decreases, the flux increases monotonically for $h \lesssim h_{c}$ and builds up rapidly at around $T_{L} \simeq 0.017$, while it becomes nonmonotonic for $h \gtrsim h_{c}$ and starts to decrease below a certain low temperature after the initial stage of increasing. (d) Entropy with $h \lesssim h_{c}$ exhibits a stable $S \simeq \frac{1}{2} \ln 2$ quasiplateau, above which the curves almost coincide; for $h \gtrsim h_{c}$, the entropy leaves a shoulderlike structure at intermediate temperature.

\section{B. Kitaev fractional liquid at finite fields}

Recently, the magnetic field effects on the Kitaev materials have raised great interest [30-33,47]. For example, the thermal quantum Hall signature has been detected in the field-induced spin liquid phase of $\alpha-\mathrm{RuCl}_{3}$ [30]. Given the fractional liquid scenario in the pure Kitaev model, it is also interesting to further study how it evolves under external fields [33]. In the following, we mainly focus on the field direction $h \|$ [001]; the effects of external fields in other directions are discussed in Appendix D, where qualitatively the same results are seen.

The finite-field entropy results are contour plotted in Fig. 3(a), with the $S$ vs $T$ curves shown explicitly in Fig. 2(d). The two isentropic lines with $S / \ln 2=0.75$ and 0.25 in Fig. 3(a) correspond to the crossover temperatures $T_{H}$ and $T_{L}$ (or $T_{L}^{\prime}$ ) very well. The intermediate- $T$ regime, which corresponds to the $\frac{1}{2} \ln 2$ entropy plateau and signifies the fractional liquid, extends to a rather wide field range regardless of whether the low- $T$ phase is an asymptotic KSL or not. The two-step release of entropy gives rise to the double-peaked specific-heat curves as illustrated in Figs. 3(b) and 4.

While $T_{H}$ remains stable as the magnetic field $h$ increases, the low- $T$ crossover temperature $T_{L}$ drops at $h=h_{c} \simeq 0.02$ and increases again as $h$ further increases [T $T_{L}^{\prime}$ in Fig. 3(b)]. As a matter of fact, $T_{L}^{\prime}$ represents a different low- $T$ scale since for $h>h_{c}$ the ground state is no longer a KSL but a topologically trivial field-induced FM phase. This can be seen evidently in Figs. 3(c) and 3(d), where the KSL is maintained 


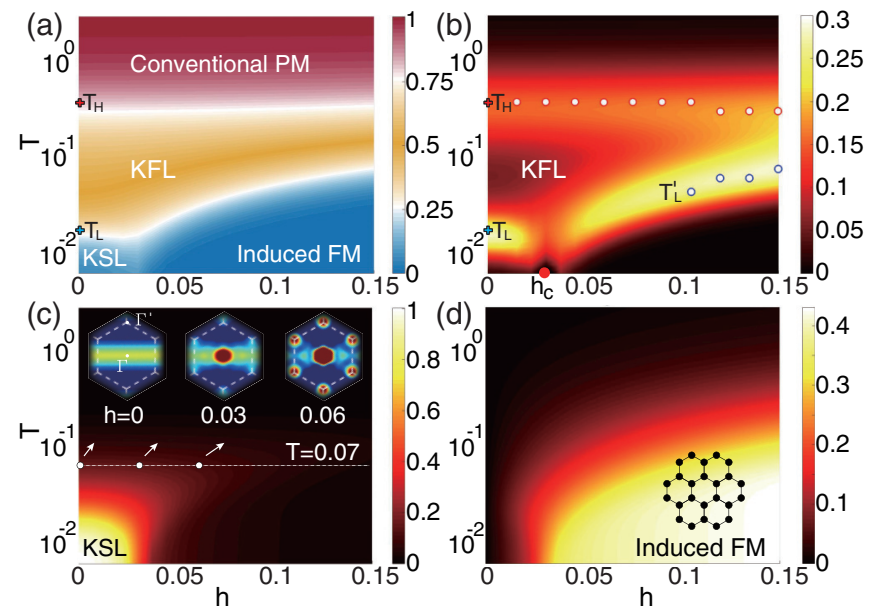

FIG. 3. Contour plot of (a) thermal entropy $S / \ln 2$ and (b) specific heat of the Kitaev model under magnetic fields $h$, where two crossover temperature scales and an intermediate Kitaev fractional liquid (KFL) regime are evident. The $T_{H}$ and $T_{L}\left(T_{L}^{\prime}\right)$ determined from (b) coincide with results from Ref. [33] (the white dots, available for $T \gtrsim 0.05$ for $h \neq 0$ ). (c) Flux $W_{P}$ and (d) magnetization $M$ are shown vs various $h$ and $T$. Three finite- $T(T=0.07)$ spin-structure factors at $h=0,0.03$, and 0.06 are presented in the inset of (c) (whose intensity color bar ranges from 0.2 to 0.5 ). A structure peak emerges at the center ( $\Gamma$ point) of the extended Brillouin zone due to the induced FM moment $M$ at $h>0$, together with a stripy background. (d) The field-induced FM phase is clearly indicated and the black dots represent the spins parallel to the [001] field in the inset.

for $h \lesssim h_{c}\left[W_{P} \neq 0\right.$; see also Fig. 2(c)], while the field-induced moment $M=\left(\frac{1}{N} \sum_{i}\left\langle S_{i}^{z}\right\rangle\right) \neq 0$ becomes significant only for $h \gtrsim h_{c}$. Thus the transition field $h_{c} \simeq 0.02$ is in very good agreement with previous studies [48,49], although [111] fields are usually considered.

\section{Spin correlation and structure factor}

In the robust fractional liquid regime, we again observe extremely-short-range and bond-directional spin correlations that characterize this exotic finite- $T$ quantum state even under external fields. In Fig. 2(b) we plot the spin correlations

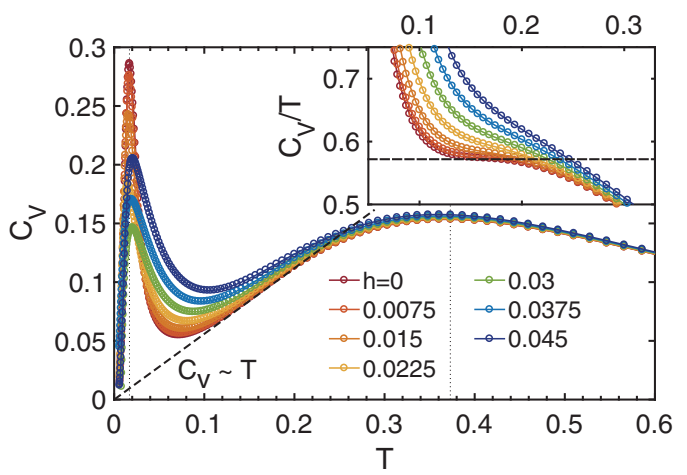

FIG. 4. Specific heat $C_{V}$ under various magnetic fields $h$. The linear- $T$ dependence of $C_{V}$ at intermediate temperature is evident as indicated by the black dashed line, which also underlines the quasiplateau in the $C_{V} / T$ curves plotted in the inset. $\left\langle S_{i}^{z} S_{j}^{z}\right\rangle-M^{2}$ with the field-induced uniform background subtracted. Although the longer-range correlations rise up at lower temperature and spoil the extremely short correlation length inherited from the pure Kitaev model, we nevertheless can observe that the $d_{z}$ correlation is predominant over a very wide range of intermediate temperatures.

Correspondingly, we compute the static spin-structure factor from the equal-time correlation function

$$
S^{\gamma}(q)=\sum_{j}\left\langle S_{i_{0}}^{\gamma} S_{j}^{\gamma}\right\rangle_{\beta} e^{i q\left(r_{j}-r_{0}\right)},
$$

where $r_{0}$ is the position vector of the fixed reference site $\left(i_{0}\right)$ and $j$ (and thus vector $r_{j}$ ) runs over the lattice sites. We observe in Fig. 3(c) a stripy structure factor at intermediate temperature for both $h=0$ and $h \neq 0$, resembling that in the KSL ground state [17,50,51]. For $h>0$, there exists an additional bright spot at the $\Gamma$ point, which represents the field-induced FM background in the system and can be observed clearly at intermediate temperature. Besides the twopoint correlation, from Figs. 2(c) and 3 we observe that the $\mathbb{Z}_{2}$ flux operator $W_{P}$ remains small at intermediate temperature, suggesting that the gauge fluxes can still flip freely under various fields in the fractional liquid regime.

\section{Linear- $T$ specific heat in the Majorana metal}

In the Kitaev fractional liquid, the itinerant Majorana fermions couple to the thermally fluctuating fluxes and lead to a finite density of states emerging at the Fermi level, when averaged over the disordered flux sectors, and can be characterized by a linear $T$-dependent behavior of the specific heat $C_{V}$ [26]. It is therefore of great interest to check if this intermediate- $T$ Majorana metal state could persist at finite external fields, by examining the specific-heat behaviors.

In Fig. 4 we show the XTRG results of $C_{V}$ that indeed exhibit linear behaviors in the lower part of the intermediate- $T$ regime, in excellent agreement with previous QMC results at zero field [26], and thus extend the conclusion there to a finite range of fields. As temperature decreases further, for $h \lesssim h_{c}$ the $C_{V}$ curve deflects the linear- $T$ behavior slightly above $T_{L}$ (or $T_{L}^{\prime}$ ); when the fields $h \gtrsim h_{c}$, the low- $T$ scale $T_{L}^{\prime}$ increases as $h$ increases, resulting in a reduction of the temperature range showing a metallic specific heat. We reveal this behavior more clearly in the inset of Fig. 4, where a plateau appears in $C_{V} / T$ for $h$ up to $h_{c} \simeq 0.02$ and then turns into a shoulderlike structure for larger fields up to $h=0.045$, reminiscent of the itinerant Majorana metal.

\section{EMERGENT CURIE LAW IN THE FRACTIONAL LIQUID}

The linear- $T$ specific-heat scaling constitutes an exotic thermodynamics of the Kitaev fractional liquid. However, both the itinerant Majorana fermion and $\mathbb{Z}_{2}$ flux have contributions in the specific heat and thus the Majorana metallic behavior is masked by the entropy release of flux freezing at low temperature near $T_{L}$. Moreover, in experiments, there are contributions other than the Majorana fermions, e.g., magnon excitations on top of the long-range magnetic order and the excess phonon contribution not fully deducted, that may enter 


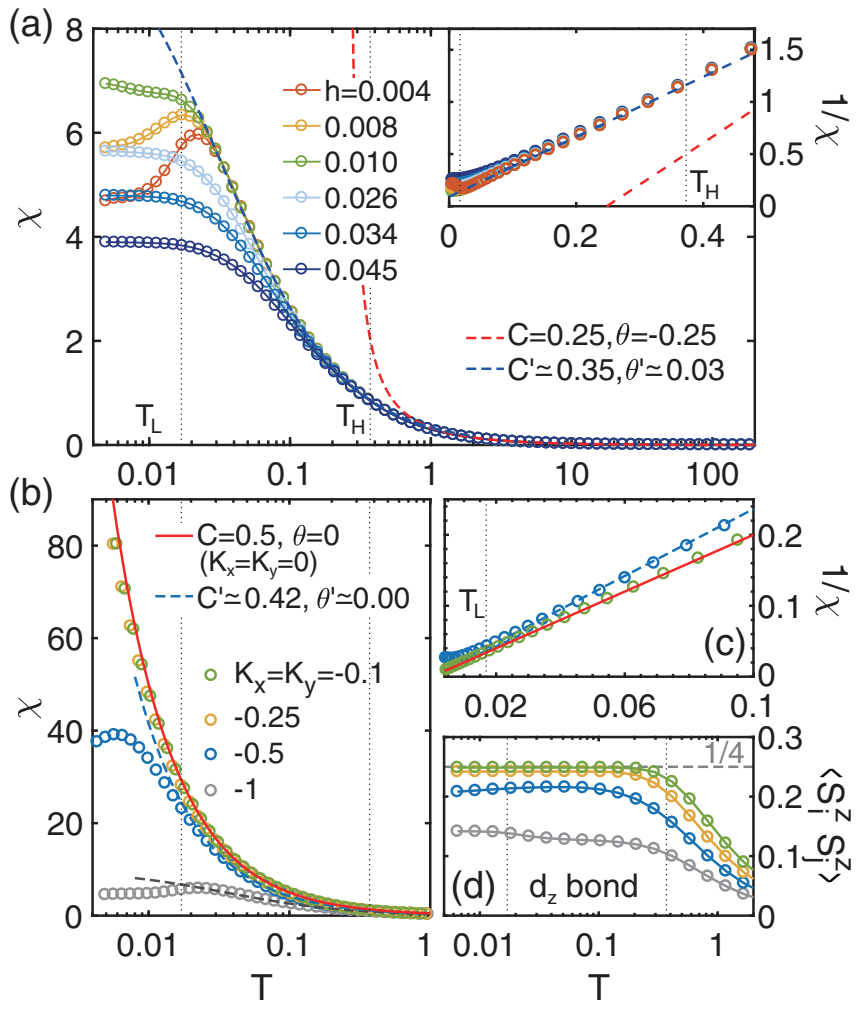

FIG. 5. (a) Uniform magnetic susceptibility $\chi$ under various fields $h$, with $1 / \chi$ plotted in the inset. The Curie-Weiss fittings in the high- and intermediate- $T$ regimes are indicated by the red and blue dashed lines, respectively. The high- $T$ behavior can be fitted well by $1 /(4 T-J)$, and immediately below $T \simeq T_{H}$ there emerges a second Curie behavior. (b) Zero-field susceptibility of the anisotropic model with $K_{x}=K_{y} \neq K_{z}$, where the emergent Curie behaviors are observed in a wider temperature range. Also presented are the corresponding (c) $1 / \chi$ fittings and (d) spin correlations on the $d_{z}$ bond.

the intermediate- $T$ specific-heat data and thus mask the expected universal linear- $T$ behavior.

Therefore, an in-depth analysis of other readily accessible thermodynamic measurements in experiments is called for that reflects more of the intrinsic magnetic properties of the system, i.e., the susceptibility $\chi$. From the analysis, we reveal that a universal Curie-law behavior emerges in the intermediate- $T$ susceptibility that is robust against non-Kitaev terms and originates from the fractionalization of spins.

\section{A. Magnetic susceptibility in the isotropic and anisotropic Kitaev models}

As a direct characterization of unconventional paramagnetism of the fractional liquid, in Fig. 5(a) we show the susceptibility of the isotropic FM Kitaev model under various magnetic fields. For small fields $h \lesssim h_{c}$, the susceptibility data collapse into a universal Curie form $\chi=\frac{C^{\prime}}{T}$ in the fractional liquid regime, further consolidated by plotting $1 / \chi$ vs $T$ in the inset of Fig. 5(a). This unexpected universal Curie behavior extends across the fractional liquid regime, spanning a very wide temperature range of about one order of magnitude $(T \sim$ 0.03-0.3), and constitutes an emergent phenomenon distinct from conventional paramagnetism at high temperature. The latter corresponds to a Curie constant $C=S(S+1) / 3=\frac{1}{4}$ related to the intrinsic moment of spin $S=\frac{1}{2}$, while the emergent Curie constant $C^{\prime} \approx \frac{1}{3}$ [see Fig. 5(a)] in the fractional liquid regime, suggesting the presence of spin correlations that renormalize the effective magnetic moments.

Besides the isotropic case, we turn to the anisotropic Kitaev model and also find emergent Curie behaviors in Figs. 5(b) and 5(c) that can extend down to even lower temperatures. The Curie-Weiss fittings give $C^{\prime} \approx 0.50$ for $K_{x, y}=-0.1$ and -0.25 , whose susceptibility is already very close to the ensemble of isolated FM spin pairs [red lines in Figs. 5(b) and 5(c)], and the Curie constant is close to twice a single spin $\frac{1}{2}$, i.e., $C=\frac{1}{2}$. The emergent Curie susceptibility there can extend down to temperatures significantly lower than $T_{L}$ (of the isotropic case), due to the reduction of flux gap. Therefore, starting from the trivial $K_{x, y}=0$ limit, as we turn on the FM coupling $K_{x, y}$ in the system, the Curie behavior remains, although the temperature range gets narrowed and the Curie constant becomes modified. For example, with $K_{x, y}=$ -0.5 , there also exists a Curie-law regime with $C^{\prime}$ reduced to 0.42 , as indicated by the blue dashed lines in Figs. 5(b) and 5(c). This universal Curie scaling extends all the way to the isotropic point, implying that spins can be pair flipped nearly freely in the intermediate- $T$ regime.

In fact, the emergent Curie behaviors can be observed as long as the spin correlations $\left\langle S_{i}^{z} S_{j}^{z}\right\rangle$ are mostly restricted within the $d_{z}$ bond [see Figs. 2(a) and 2(b)] and in a quasiplateau vs $T$ at intermediate temperature shown in Fig. 5(d). We note that, according to the perturbation theory, the $\mathbb{Z}_{2}$ fluxes remain approximately conserved and spin correlations beyond the $d_{z}$ bonds only appear at the $h_{c}^{2}$ order. At the same time, $\mathbb{Z}_{2}$ fluxes should be flippable in a nearly free fashion, which can be observed as $W_{P} \ll 1$ in Fig. 2(c). These conditions are satisfied even when perturbative external fields are applied, which gives rise to the intermediate- $T$ Curie law and will be elaborated in Sec. IV B.

\section{B. Fluctuating fluxes and diverging susceptibility}

The diverging susceptibility at intermediate temperature reflects the strongly fluctuating fluxes in the fractional liquid below $T_{H}$, and deviation occurs when the flux starts to freezeout at about $T_{L}$. To see this, we exploit the Kubo formula of susceptibility (see Appendix E) and assume that $M=0$ in the fractional liquid for the sake of discussion,

$$
\chi=\sum_{j} \int_{0}^{\beta}\left\langle S_{i_{0}}^{z}(\tau) S_{j}^{z}\right\rangle_{\beta} d \tau,
$$

where $S_{i_{0}}^{z}(\tau)=e^{\tau H} S_{i_{0}}^{z} e^{-\tau H}$ and $j$ runs over NN sites of $i_{0}$ by the $d_{z}$ bond (as well as $i_{0}$ itself) in the fractional liquid regime due to the extremely-short-range correlations. As illustrated in Fig. 1, the spin operator $S_{j}^{z}$ flips a pair of fluxes on two neighboring hexagons (in purple) and changes the flux configuration of energy eigenstate. After evolving along the imaginary time of $\tau, S_{i_{0}}^{z}$ can flip the $\mathbb{Z}_{2}$ fluxes back and thus restore the flux configurations, which results in a nonzero correlation.

We denote the energy eigenstates by $\left|E_{\left\{W_{P}\right\}}^{n}\right\rangle$, where $\left\{W_{P}\right\}$ represents the flux configurations and $n$ labels the individual 
states within the $\left\{W_{P}\right\}$ sector. By inserting the orthonormal basis, we arrive at the Lehmann spectral representation

$$
\begin{aligned}
\left\langle S_{i_{0}}^{z}(\tau) S_{j}^{z}\right\rangle_{\beta}= & \sum_{\left\{W_{P}\right\}, n} \sum_{n^{\prime}} e^{-\beta E_{\left\{W_{P}\right\}}^{n}} e^{-\tau \Delta_{n,\left\{W_{P} ; n^{\prime}, \mid W_{P}^{\prime}\right\}}} \\
& \times\left\langle E_{\left\{W_{P}\right\}}^{n}\left|S_{i_{0}}^{z}\right| E_{\left\{W_{P}^{\prime}\right\}}^{n^{\prime}}\right\}\left\langle E_{\left\{W_{P}^{\prime}\right\}}^{n^{\prime}}\left|S_{j}^{z}\right| E_{\left\{W_{P}\right\}}^{n}\right\rangle,
\end{aligned}
$$

where $\left|E_{\left\{W_{p}^{\prime}\right\}}^{n^{\prime}}\right\rangle$ represents a state $n^{\prime}$ in the flux-flipped sector $\left\{W_{P}^{\prime}\right\}$. Since the itinerant Majorana fermions are weakly coupled to the flux at intermediate temperature [26] and thus the excitation energy is close to the flux gap, $\Delta_{n,\left\{W_{P}\right\} ; n^{\prime},\left\{W_{P}^{\prime}\right\}}=$ $\left|E_{\left\{W_{P}^{\prime}\right\}}^{n^{\prime}}-E_{\left\{W_{P}\right\}}^{n}\right| \sim T_{L} \ll T \equiv 1 / \beta$. Therefore, the decay factor $e^{-\tau \Delta_{n,\left\{W_{P} ;: n^{\prime},\left\{W_{P}^{\prime}\right\}\right.}} \simeq 1$, which is not surprising as different flux sectors are nearly degenerate in the fractional liquid regime, as evidenced by $W_{P} \ll 1$ [see, e.g., Fig. 2(c)].

Consequently, $\left\langle S_{i_{0}}^{z}(\tau) S_{j}^{z}\right\rangle_{\beta}$ is virtually $\tau$ independent at intermediate temperature and can be approximated as $\left\langle S_{i_{0}}^{z} S_{j}^{z}\right\rangle_{\beta}$ in Eq. (3), thus recovering the conventional susceptibility formula

$$
\chi=\frac{1}{T} \sum_{j}\left\langle S_{i_{0}}^{z} S_{j}^{z}\right\rangle_{\beta},
$$

which holds only for $T \gtrsim T_{L}$. As observed in Figs. 2(a) and 5(d), the correlation $\left\langle S_{i_{0}}^{z} S_{j}^{z}\right\rangle_{\beta}$ exhibits a quasiplateau vs temperatures between $T_{L}$ and $T_{H}$, resulting in a $1 / T$-divergent susceptibility that accounts for the emergent Curie law in the fractional liquid regime. On the other hand, in the KSL regime at $T \lesssim T_{L}$, although the correlation length remains extremely short there, the (flux) excitation energy $\Delta_{n,\left\{W_{P}\right\} ; n^{\prime},\left\{W_{P}^{\prime}\right\}} \sim T_{L} \gtrsim$ $T$ leads to an exponentially suppressive factor in $\tau$ and thus a nondivergent susceptibility $\chi$ below $T_{L}$ in Fig. 5 .

Above we discussed the emergent susceptibility scaling in the FM Kitaev model and below we briefly discuss the case of the AF model, with the details deferred to Appendix F. For the AF Kitaev model, the spin correlations are also extremely short ranged and their absolute values are identical to those of the FM case, but with opposite sign (AF correlation). Therefore, the emergent Curie law is absent in the uniform susceptibility $\chi$ of the AF Kitaev model, while it appears instead in the stagger susceptibility. This can be understood intuitively as follows: One can flip simultaneously the spins on one of the two sublattices on the honeycomb lattice, turning the AF Kitaev model into an FM one, and the staggered susceptibility $\chi_{s}$ corresponds to the uniform $\chi$ exhibiting universal scaling at intermediate temperature. Such an interesting correspondence between the FM Kitaev model under uniform field and the AF model under staggered field has been noticed in other studies under different circumstances [52]. Our results indicate a sensitive way to determine the sign of Kitaev coupling via analyzing the susceptibility measurements.

\section{THERMAL TENSOR NETWORK SIMULATIONS OF THE EXTENDED KITAEV MODELS}

Now we switch to the XTRG simulations of the extended Kitaev models with the Heisenberg and $\Gamma$ interactions, which hinders other quantum many-body methods like QMC by the sign problem. The Heisenberg coupling is proposed to be relevant in the Kitaev material $\mathrm{Na}_{2} \mathrm{IrO}_{3}[13,15]$ and the Kitaev-Heisenberg model has thus been extensively studied for both the ground-state $[13,16,48,50,53]$ and finite- $T$ properties [54,55]. On the other hand, in $\alpha-\mathrm{RuCl}_{3}$ there exists a symmetric off-diagonal exchange, the so-called $\Gamma$ term $[41,49,51,56]$, which turns out to be important non-Kitaev interactions. In this section we explore the Kitaev-Heisenberg (on YC $4 \times 6 \times 2$ cylinders) and Kitaev- $\Gamma($ YC $4 \times 4 \times 2$ ) models [see Eq. (1)] at finite temperature, from which we see that although the low-temperature fate of the Kitaev model may be drastically affected by the Heisenberg and $\Gamma$ terms, the behavior at high and intermediate temperatures, especially in the Kitaev fractional liquid regime, remains qualitatively unchanged.

\section{A. Fractional thermal entropy and specific-heat behaviors}

We start with the thermal entropy and specific-heat calculations of the Kitaev-Heisenberg and Kitaev- $\Gamma$ models and show the results in Figs. 6 and 7. Between the two entropy-release steps [Figs. 7(c) and 7(d)], there exists an extended regime with thermal entropy $S \simeq \frac{1}{2} \ln 2$ [Figs. 6(a) and 6(b)] for both models, which indicates the existence of Kitaev fractional liquid at intermediate temperature. The $\frac{1}{2}$-entropy quasiplateau remains evident until Heisenberg coupling $J \lesssim 0.16$ and for a $\Gamma$ interaction of $\Gamma \lesssim 0.06$. Beyond this $\Gamma$ or $J$ range, a shoulderlike feature with slow entropy release can still be recognized in both models. In Figs. 6(a) and 6(b) the crossover temperature scales $T_{H}$ and $T_{L}\left(T_{L}^{\prime}\right)$ can be determined from the isentropic lines of $S / \ln 2=0.75$ and 0.25 , which coincide with the higher and lower crossover temperatures in the double-peaked specific-heat curves [see Figs. 7(a) and 7(b) and also Appendix G].

In Figs. 7(a) and 7(b) we show that the linear- $T$ behavior of specific heat also survives in the presence of a finite $J$ or $\Gamma$ interaction, which also can be recognized from the $C_{V} / T$ plots in the insets. This universal specific-heat behavior suggests the emergence of Majorana-metal states in the fractional liquid regime. Nevertheless, similar to what was observed in Fig. 4, the $J$ and $\Gamma$ interactions can shift the flux excitations as well as other low-energy spin fluctuation modes towards higher temperatures and thus may mask the linear- $T C_{V}$, leaving eventually only a shoulderlike remnant in the insets of Figs. 7(a) and 7(b).

The finite- $T$ properties can sensitively reflect the quantum phase transition in the ground state. In Fig. 6(a), $T_{L}\left(T_{L}^{\prime}\right)$ is nonmonotonic vs $J$ : For $J \lesssim J_{c} \simeq 0.12, T_{L}$ represents a crossover from the KSL to the fractional liquid, while for $J>J_{c}$, the stripe antiferromagnetic (SAF) order sets in below $T_{L}^{\prime}$, where correlations along $d_{x}, d_{x+y}$, etc, also increase rapidly as shown in Fig. 6(c). Therefore, we infer from the finite- $T$ results that the Kitaev-Heisenberg model undergoes a transition from the KSL to SAF phase at around $J_{c} \simeq 0.12$, consistent with previous ground-state studies $[13,53,56]$.

\section{B. Bond-directional spin correlation and stripy structure factor}

From the spin-correlation data shown in Figs. 6(c) and 6(d), we emphasize that the two models share common features in the Kitaev fractional liquid regime. First, the spin 

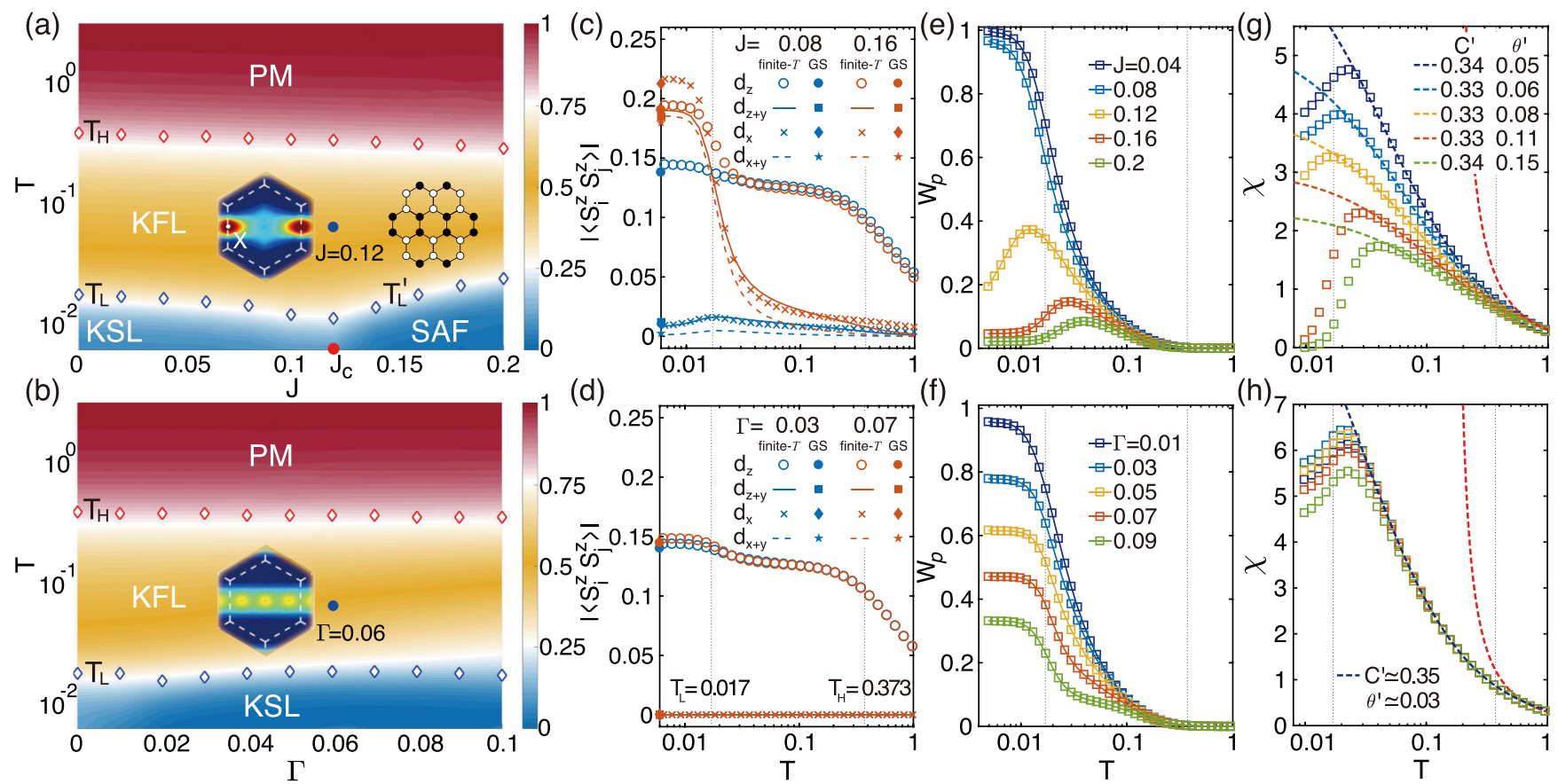

FIG. 6. Phase diagram of (a) the Kitaev-Heisenberg and (b) the Kitaev- $\Gamma$ model obtained by entropy $S / \ln 2$, where the white diamonds indicate the crossover temperature determined from the double-peaked specific heat. The insets show the $T=0.07$ static structure factors of (a) $J=0.12$ and (b) $\Gamma=0.06$ in the extended Brillouin zone. The XTRG results of the correlations $\left\langle S_{i}^{z} S_{j}^{z}\right\rangle$ along four NN bonds with (c) $J=0.08$ and 0.16 and (d) $\Gamma=0.03$ and 0.07 all converge at the lowest temperature to the GS values calculated by the DMRG method. For the Kitaev-Heisenberg case with $J=0.08$ and the Kitaev- $\Gamma$ case, $d_{z}$ correlations arise at around $T_{H}$ and stay predominate across the whole temperature range; for the $J=0.16$ case, all correlations increase abruptly at the low-temperature scale, indicating the onset of SAF order. (e) and (f) Flux $W_{P}$ with various $J$ or $\Gamma$ and (g) and (h) magnetic susceptibility $\chi$ and the Curie-Weiss fittings for two models. The $\chi$ data are computed under a small uniform field $h=0.002$.

correlation $\left\langle S_{i}^{z} S_{j}^{z}\right\rangle$ remains extremely short ranged and mostly present on the $d_{z}$ bonds. This is consistent with a perturbationtheory analysis: Except on the $d_{z}$ bonds, spin correlations are suppressed because $\mathbb{Z}_{2}$ fluxes are approximately conserved. Furthermore, such correlations appear at the first order in $J$ but only at the second order in $\Gamma$, consistent with the fact that $J$ has a larger effect on spin correlations than $\Gamma$, as shown in Figs. 6(c) and 6(d). This prominent spin-correlation feature consequently leads to the stripy structure factor in the insets of Figs. 6(a) and 6(b).

Under the Heisenberg interactions, we observe in the inset of Fig. 6(a) a clear structure peak at the $X$ point, which indicates the enhanced short-range SAF correlation, with the classical spin pattern shown on the right-hand side. Besides the SAF peak, we notice that there exists a stripy structure background which is related to the predominant bond-directional NN correlations at intermediate temperature. On the other hand, in Fig. 6(b), for the Kitaev- $\Gamma$ model, the low-temperature scale $T_{L}$ changes only slightly. The KSL-like stripy spin structure can be observed with no clear signature of spin ordering, which, together with the nonzero fluxes $W_{P} \neq 0$, at low temperature in Fig. 6(f), suggests that the low- $T$ quantum state remains in the asymptotic KSL phase at least for $\Gamma \lesssim 0.1$, in agreement with recent ground-state studies of the Kitaev- $\Gamma$ model $[49,56]$.

\section{Universal susceptibility scaling}

At intermediate temperature, we again observe bonddirectional spin correlations that are mostly restricted within the NN sites [see Figs. 6(c) and 6(d) and discussion above], as well as the strongly fluctuating flux [Figs. 6(e) and 6(f)] under an extended range of $J$ or $\Gamma$ interactions. Following the discussion in the pure Kitaev model, we expect an emergent Curie law of susceptibility in the fractional liquid, and this is indeed observed in Figs. 6(g) and 6(h). Although the temperature window gradually narrows as $J$ or $\Gamma$ increases, the universal susceptibility scaling can be very clearly identified across the intermediate- $T$ regime, regardless of whether the ground state is a KSL or semiclassically ordered.

Nevertheless, there are still some differences in the susceptibility scalings between the Kitaev-Heisenberg and Kitaev- $\Gamma$ models. At the temperature above $T_{L}$, the susceptibility curves in the Kitaev- $\Gamma$ model collapse into the same universal behavior, with $C^{\prime} \approx \frac{1}{3}$ (thus $C^{\prime} / C \approx 1.33$, the same as in the pure Kitaev model) and with the same small $\theta^{\prime}$. This is in contrast to the case of the Kitaev-Heisenberg model, where although $C^{\prime} \simeq \frac{1}{3}$ remains, the Curie-Weiss temperature $\theta^{\prime}$ grows as $J$ increases, which suggests that the fluxes are no longer free but are coupled with each other since the Heisenberg coupling $J$ introduces longer-range correlations to the system [Fig. 6(c)]. Accordingly, in Fig. 6(e), the gauge flux for $J \lesssim J_{c}$ behaves very similarly to that of the pure Kitaev model, i.e., $W_{P}$ freezes 


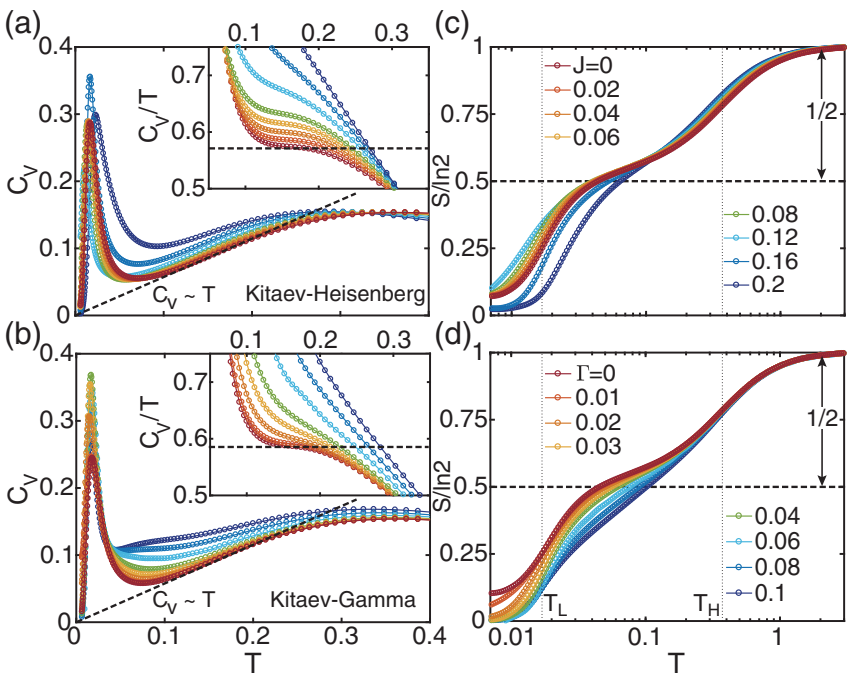

FIG. 7. Specific heat $C_{V}$ vs $T$ for the (a) Kitaev-Heisenberg and (b) Kitaev- $\Gamma$ models with various coupling strengths. The black dashed lines indicate the linear $T$ dependence of $C_{V}$ at intermediate temperature and the corresponding $C_{V} / T$ data are shown in the insets. With Heisenberg interactions in (a), the $C_{V} / T$ plateau is apparent for $J \lesssim 0.08$ and leaves a shoulderlike structure until $J_{c} \simeq 0.12$; for the $\Gamma$ terms this plateau and its remnant signature are clear for $\Gamma \lesssim 0.04$. (c) and (d) Thermal entropy results of both models. Around the stable high- $T$ scale $T_{H}$, the entropy curves almost collapse for various $J$ and $\Gamma$ strengths and consequently leave a quasiplateau with $S \simeq \frac{1}{2} \ln 2$.

at around $T_{L}$, while it gets suppressed at low temperature for $J>J_{c}$

Overall, we observe that the emergent Curie law of susceptibility scaling appears to be robust against the couplings beyond the pure Kitaev model at intermediate temperature, which is found to be suppressed only when the finite flux gap or the longer-range correlation induced by the non-Kitaev terms takes effect at some low temperature. Therefore, such universal susceptibility scaling can serve as a useful tool probing the free $\mathbb{Z}_{2}$ flux and thus fractionalization in the Kitaev materials.

\section{DIAGNOSIS OF THE KITAEV FRACTIONAL LIQUID}

\section{A. Kitaev paramagnetism in the Kitaev materials}

Thermodynamic features, including the double-peaked specific-heat curve, the linear- $T$ behavior, and the fractional thermal entropy $S$ integrated from $C_{V} / T$, have been exploited in detecting finite- $T$ fractionalization in the Kitaev materials [18,24,57-59]. However, the magnetic response to external fields, the susceptibility $\chi$, has been much less analyzed, except for the high- $T$ behaviors in the Kitaev materials. With insight from the emergent intermediate- $T$ Curie susceptibility from the above model studies, we revisit the experimental $\chi$ data of two prominent Kitaev materials, i.e., $\mathrm{Na}_{2} \mathrm{IrO}_{3}$ [14] and $\alpha-\mathrm{RuCl}_{3}$ [57], which are believed to realize the Kitaev model under the Heisenberg and/or $\Gamma$ interactions.

In Fig. 8 we analyze the in-plane and out-of-plane susceptibility data of both materials, where the emergent
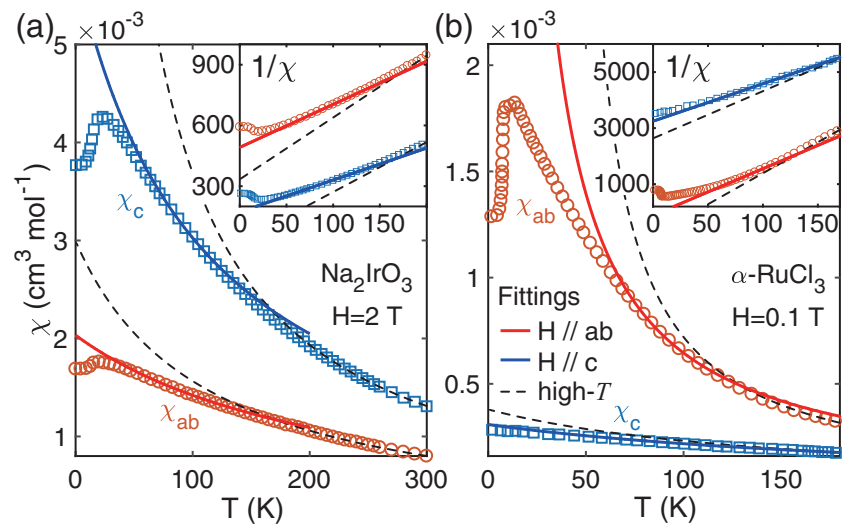

(c)

\begin{tabular}{lcccccccccc}
\hline \hline & $\mathrm{C}_{\mathrm{ab}}$ & $\theta_{\mathrm{ab}}$ & $\mathrm{C}_{\mathrm{c}}$ & $\theta_{\mathrm{c}}$ & $\mathrm{C}_{\mathrm{ab}}^{\prime}$ & $\theta_{\mathrm{ab}}^{\prime}$ & $\mathrm{C}_{\mathrm{c}}^{\prime}$ & $\theta_{\mathrm{c}}^{\prime}$ & $\mathrm{C}_{\mathrm{ab}}^{\prime} / \mathrm{C}_{\mathrm{ab}}$ & $\mathrm{C}_{\mathrm{c}}^{\prime} / \mathrm{C}_{\mathrm{c}}$ \\
\hline $\mathrm{Na}_{2} \mathrm{IrO}_{3}$ & 0.33 & -110 & 0.41 & -10 & 0.47 & -232 & 0.63 & -110 & 1.42 & 1.54 \\
$\alpha-\mathrm{RuCl}_{3}$ & 0.045 & 37 & 0.059 & -156 & 0.060 & 7 & 0.075 & -242 & 1.33 & 1.27 \\
\hline
\end{tabular}

FIG. 8. Experimental $\mathrm{Na}_{2} \mathrm{IrO}_{3}[14]$ and $\alpha-\mathrm{RuCl}_{3}$ susceptibility data [57] and their Curie-Weiss fittings at both (a) high and (b) intermediate temperatures under fields $H \| a b$ (orange circles) and $H \| c$ (blue squares), with inverse susceptibility plotted in the insets. (c) Fitted Curie constants ( $C$ for high and $C^{\prime}$ for intermediate $T$ ) and the Curie temperatures (corresponding $\theta$ and $\theta^{\prime}$ ), for both the $c$ and $a b$ directions, are listed in the table.

Curie behaviors can be observed right below certain hightemperature scales, i.e., $T_{H}^{\mathrm{Ir}} \simeq 120 \mathrm{~K}$ and $T_{H}^{\mathrm{Ru}} \simeq 110 \mathrm{~K}$ for $\mathrm{Na}_{2} \mathrm{IrO}_{3}$ and $\alpha-\mathrm{RuCl}_{3}$, respectively. Here $T_{H}^{\mathrm{Ir}}$ and $T_{H}^{\mathrm{Ru}}$ are in agreement with the higher crossover temperatures in the magnetic specific-heat curves of two materials. For example, in $\mathrm{Na}_{2} \mathrm{IrO}_{3}$ the higher specific-heat peak is located at around $110-120 \mathrm{~K}[17,18]$, which is in very good agreement with the $T_{H}^{\mathrm{Ir}}$ in Fig. 8(a). Similarly, the high- $T$ entropy is rapidly released at around $100-110 \mathrm{~K}$ in $\alpha-\mathrm{RuCl}_{3}$ [24,57,59], also coinciding with $T_{H}^{\mathrm{Ru}}$ in Fig. 8(b).

Through Curie-Weiss fittings of the intermediate- $T$ susceptibility results in Fig. 8, we find the the ratios between intermediate- and high- $T$ Curie constants $C^{\prime} / C \sim 1.3$ in both materials (and either within the $a b$ plane or parallel to the $c$ axis), suggesting the existence of the Kitaev fractional liquid in these two Kitaev materials. The emergent diverging susceptibility at intermediate temperature is very sensitive to the sign of the Kitaev interaction $K$, and for the AF case $(K>0)$ a convex uniform susceptibility curve is exhibited without any emergent Curie behavior at intermediate temperature (Appendix F). Therefore, the analysis in Fig. 8 suggests the FM Kitaev interactions in both compounds $\mathrm{Na}_{2} \mathrm{IrO}_{3}$ and $\alpha-\mathrm{RuCl}_{3}$, in agreement with previous theoretical $[10,38,60,61]$ and experimental [40] estimates.

Meanwhile, in Fig. 8 we observe that the intermediate Curie-Weiss behaviors deviate at the lower temperature $T_{L}^{\mathrm{Ir}} \simeq$ $50-55 \mathrm{~K}$ and $T_{L}^{\mathrm{Ru}} \simeq 60-65 \mathrm{~K}$. Therefore, in these two materials there are fractional liquid regimes stretching appreciable temperature ranges as wide as $65-70 \mathrm{~K}\left(\mathrm{Na}_{2} \mathrm{IrO}_{3}\right)$ and $45-$ $50 \mathrm{~K}\left(\alpha-\mathrm{RuCl}_{3}\right)$, respectively. In addition, by comparing Fig. 8(a) to Fig. 6(g), we find a wider intermediate- $T$ range for the emergent Curie-Weiss in $\mathrm{Na}_{2} \mathrm{IrO}_{3}$. This resembles that of the Kitaev-Heisenberg model, where universal scaling 

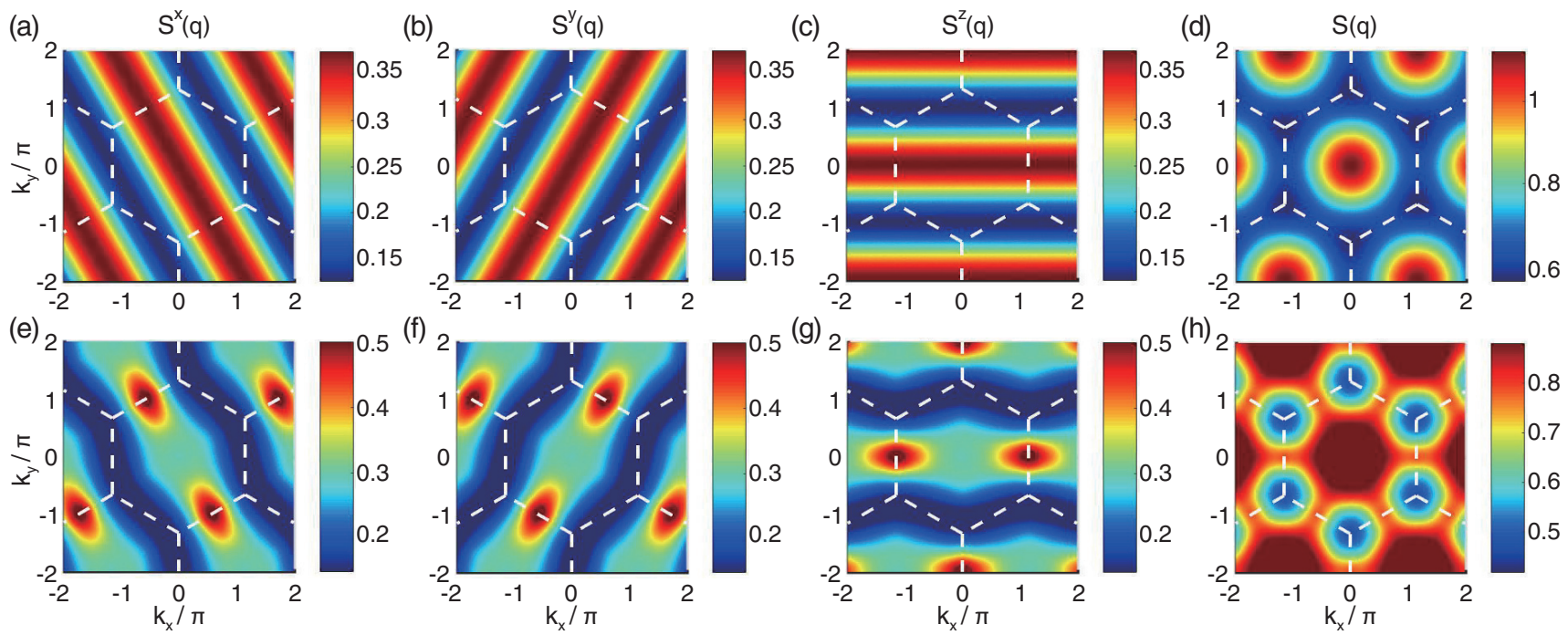

FIG. 9. Static spin-structure factors $S^{\gamma}(q)$ calculated from the equal-time spin-correlation functions [see Eq. (2)] at an intermediate temperature $T \simeq 0.15$ : (a)-(d) pure Kitaev model results of three spin-structure factors $S^{x, y, z}(q)$ as well as their sum $S(q)$ and (e)-(h) results of the Kitaev-Heisenberg model with $J=0.14$.

deviates at around the susceptibility peak temperature. This observation suggests that indeed the Heisenberg-type interaction can play an important role in this material [13]. On the other hand, in Fig. $6(\mathrm{~g})$ the $\Gamma$ term deflects the Curie-Weiss behavior way before the susceptibility peaks; this bears a resemblance to the case of $\alpha-\mathrm{RuCl}_{3}$ shown in Fig. 8(b), supporting thus the proposal that the $\Gamma$ term (together with other interactions) is particularly relevant in this material [42,62].

Overall, through analysis of the magnetic susceptibility data of $\mathrm{Na}_{2} \mathrm{IrO}_{3}$ and $\alpha-\mathrm{RuCl}_{3}$, we find a universal susceptibility scaling that may be ubiquitous in other members of the intriguing family of Kitaev materials and can serve as a sensitive thermodynamic signature of the Kitaev fractional liquid. In addition, the susceptibility scaling indicates the existence of ferromagnetic Kitaev interactions, together with other non-Kitaev couplings, in the two prominent Kitaev materials.

\section{B. Spin-correlation diagnostics of the Kitaev fractional liquid}

The short-range correlated Kitaev fractional liquid leaves unique features in the spin correlations and thus structure factors. At intermediate temperature, the nonzero correlation $\left\langle S_{i}^{\gamma} S_{j}^{\gamma}\right\rangle$ is restricted within the $\mathrm{NN} \gamma$ bond, with the rest of the correlations negligibly small even under magnetic fields and non-Kitaev interactions, as shown in Figs. 2(a), 2(b), 6(c), and 6(d). Correspondingly, in Figs. 3(c), 6(a), and 6(b) we see that this peculiar bond directional correlation pattern results in a stripylike background in the $z$-direction spin-structure factors $S^{z}(q)$.

In fact, this stripy spin structure also appears in the structure factors $S^{\gamma}(q)$ with $\gamma=x, y$, and the direction of the stripe is coincides with the associated spin components $\gamma$. As shown in Figs. 9(a) and 9(e), the $S^{x}(q)$ stripe extends from the top left to the bottom right, which rotates counterclockwise by $120^{\circ}$ when switched to $S^{y}(q)$ [Figs. 9(b) and 9(f)], followed by another $120^{\circ}$ rotation to $S^{z}(q)$ in Figs. 9(c) and $9(\mathrm{~g})$. Note that the spin-structure factor results in Fig. 9 are evaluated at an intermediate temperature $T \simeq 0.15$ for the pure Kitaev model [Figs. 9(a)-9(d)] and the KitaevHeisenberg model [Figs. 9(e)-(h)]. In the latter KitaevHeisenberg case with $J=0.14$, a structure factor peak emerges, representing the semiclassical SAF correlation, which also rotates as the spin component switches. Such a rotation of the short-range correlation peak was previously observed in the spin-resolved magnetic x-ray measurements right above the ordering transition temperature $(T \simeq 17 \mathrm{~K})$ [17], which provides direct evidence of the dominant bonddirectional interactions in the Kitaev material $\mathrm{Na}_{2} \mathrm{IrO}_{3}$. Therefore, our findings in Figs. 9(e)-9(g) confirm that this experimental observation is indeed related to the presence of Kitaev interactions in the material, although the SAF order involved here is different from the zigzag type in $\mathrm{Na}_{2} \mathrm{IrO}_{3}$.

Moreover, our results in Figs. 9(d) and 9(h) also shed light on the renowned starlike spin-structure factor observed in inelastic neutron scattering measurement of $\alpha-\mathrm{RuCl}_{3}$ [23,24]. From the full structure factor $S(q)=\sum_{\gamma} S^{\gamma}(q)$, we observe in the pure Kitaev model that the superposition of the three stripes leads to an approximately rotationally symmetric shape in Fig. 9(d). By adding bright spots representing shortrange SAF correlations on top of that, we instead see a sixfold symmetric $S(q)$ in Fig. 9(h). This clearly suggests that the star-shape structure factor (with sixfold symmetry) observed in inelastic neutron scatterings on $\alpha-\mathrm{RuCl}_{3}$ can be ascribed to the coexistence of the Kitaev bond-directional correlation and short-range zigzag order. As the temperature/energy scale gradually increases, it approaches asymptotically the rotationally symmetric spin structure in Fig. 9(d), which has also been observed in experiments.

In general, we propose that the bond-directional spin correlation and related intermediate- $T$ stripy feature provide a useful tool for determining the Kitaev materials. Specifically, diffuse x-ray (for $\mathrm{Na}_{2} \mathrm{IrO}_{3}$ ) or spin-resolved neutron (for $\alpha-\mathrm{RuCl}_{3}$ ) measurement at intermediate temperature, say, $T=50-100 \mathrm{~K}$, can be exploited to observe the stripy spin 
structures $S^{\gamma}(q)$ that serves as direct evidence of the Kitaev fractional liquid.

\section{DISCUSSION AND OUTLOOK}

In conventional spin models and magnetic materials, a "crystallization" of spins occurs from the high- $T$ gaslike paramagnetic to the low- $T$ phase with long-range order. Interestingly, in the Kitaev model and related Kitaev materials, an alternative scenario is proposed where a fractionalization of spins takes place upon cooling. This gives rise to an intermediate- $T$ Kitaev fractional liquid and eventually to the low- $T$ quantum spin liquid phase with long-range entanglement. Although the finite-temperature fractionalization of quantum spins has been established [27] in the pure Kitaev model, its relevance in real Kitaev materials remains an open question. The main challenge in the way is how to perform efficient finite-temperature simulations of the extended Kitaev model with realistic interactions.

Here we clear the obstacle with the precise XTRG method and investigate the extended Kitaev model with external magnetic fields and Heisenberg and $\Gamma$ terms. A Kitaev fractional liquid regime is found at intermediate temperature and is robust to non-Kitaev interactions, regardless of whether the ground state is in a spin liquid phase or not. The exotic finite- $T$ quantum states can be characterized by universal thermodynamics, including the fractional thermal entropy, the linear- $T$ specific heat, and in particular an emergent Curie law in magnetic susceptibility. Remarkably, this universal Curie susceptibility that reflects the thermally free gauge fluxes, together with the previously recognized metallic specific-heat behaviors of itinerant Majorana fermions, each revealing one aspect of the spin fractionalization, constitutes a comprehensive thermodynamics characterization of the exotic Kitaev fractional liquid. In light of this and from analyzing the experimental susceptibility data, we find evidence of the Kitaev fractional liquid in $\mathrm{Na}_{2} \mathrm{IrO}_{3}$ and $\alpha-\mathrm{RuCl}_{3}$, which supports the ferromagnetic Kitaev interactions in the two materials and their proximity to quantum spin liquids.

As a matter of fact, the diverging susceptibility is a robust thermodynamic feature related also to the peculiar spin correlations in the fractional liquid regime. They are extremely short ranged and bond directional, thus corresponding to the stripy spin-structure factor that rotates with the spin components. Therefore, besides the magnetic susceptibility measurement, our findings also encourage further material exploration of the fractionalization using spin-resolving neutron and resonant $\mathrm{x}$-ray scatterings. Finally, our present work paves the way to the precise determination of microscopic model parameters in the Kitaev materials by analyzing thermodynamic data, which we leave for future study.

\section{ACKNOWLEDGMENTS}

The authors are indebted to Zheng-Xin Liu, Jiu-Cai Wang, Hao-Xin Wang, Andreas Weichselbaum, Wentao Jin, and Jan von Delft for insightful discussions. This work was supported by the National Natural Science Foundation of China (Grants No. 11974036, No. 11874078, No. 11834014, and
No. 11874115) and the Fundamental Research Funds for the Central Universities.

\section{APPENDIX A: EXPONENTIAL TENSOR RENORMALIZATION GROUP METHOD APPLIED TO THE EXTENDED KITAEV MODEL}

The exponential tensor renormalization group method reaches low- $T$ thermal states exponentially fast, which has been shown to be highly efficient in both critical quantum chains and various 2D lattice systems [34-36] and is exploited to study the Kitaev model of interest. To start the XTRG procedure, we need to invoke a quasi-1D mapping of the lattice model and encode the long-range interaction information in a compact matrix product operator form. For the pure Kitaev model on the YC4 geometry, the MPO has a bond dimension of $D_{H}=7$, which increases to $D_{H}=14$ for the Kitaev-Heisenberg and Kitaev- $\Gamma$ Hamiltonians.

Given the compact MPO representation of the Hamiltonian $H$, we can represent $\rho(\tau)$, initiated at a very small initial $\tau \sim$ $10^{-3}-10^{-4}$, also as an MPO conveniently and accurately (up to machine precision) via the series expansion

$$
\rho(\tau)=e^{-\tau \hat{H}}=\sum_{n=0}^{N_{c}} \frac{(-\tau)^{n}}{n !} H^{n} .
$$

Through this expansion, $H^{n}$ can also be expressed as an MPO using tensor compression techniques [63], followed by a sumand-compression procedure that finally leads to the compact MPO form of $\rho(\tau)$.

Following the XTRG idea of the exponential cooling procedure, we square the density matrix and obtain at the $n$th step

$$
\rho\left(2^{n+1} \tau\right)=\rho\left(2^{n} \tau\right) \rho\left(2^{n} \tau\right),
$$

with which we can compute the free energy at $\beta \equiv 2^{n+2} \tau$ via the thermofield double formalism

$$
f(\beta)=-\frac{1}{\beta} \ln \operatorname{Tr}\left[\rho\left(2^{\mathrm{n}+1} \tau\right) \rho\left(2^{\mathrm{n}+1} \tau\right)\right]
$$

by a bilayer tensor trace [44]. Given the free energy, other thermodynamic quantities including the specific heat, thermal entropy, magnetic susceptibility, etc., can also be computed.

We first benchmark the XTRG results with ED on a 12-site cluster (with the same width and boundary condition as YC4 geometry but with only three sites along the zigzag edge). In Fig. 10(a) the relative error of the free energy $\delta f / f$ decreases as the retained bond dimension $D$ increases, reaching a very high accuracy with $D=600$. On top of that, we show the XTRG results of specific heat computed with $D=600$, of pure Kitaev, Kitaev-Heisenberg, and Kitaev- $\Gamma$ models, and show them in Fig. 10(b), where we also find excellent agreement with ED results.

As shown in Figs. 10(c)-10(f), we compute the thermal entropy, flux expectation, specific heat, spin correlations, and magnetic susceptibility and compare our XTRG results with the QMC data taken from Refs. [27,29]. Despite the very different system sizes, i.e., the YC4 cylinder vs the $L=20$ torus, excellent agreement between two methods can be observed at high and intermediate temperatures, covering the fractional 

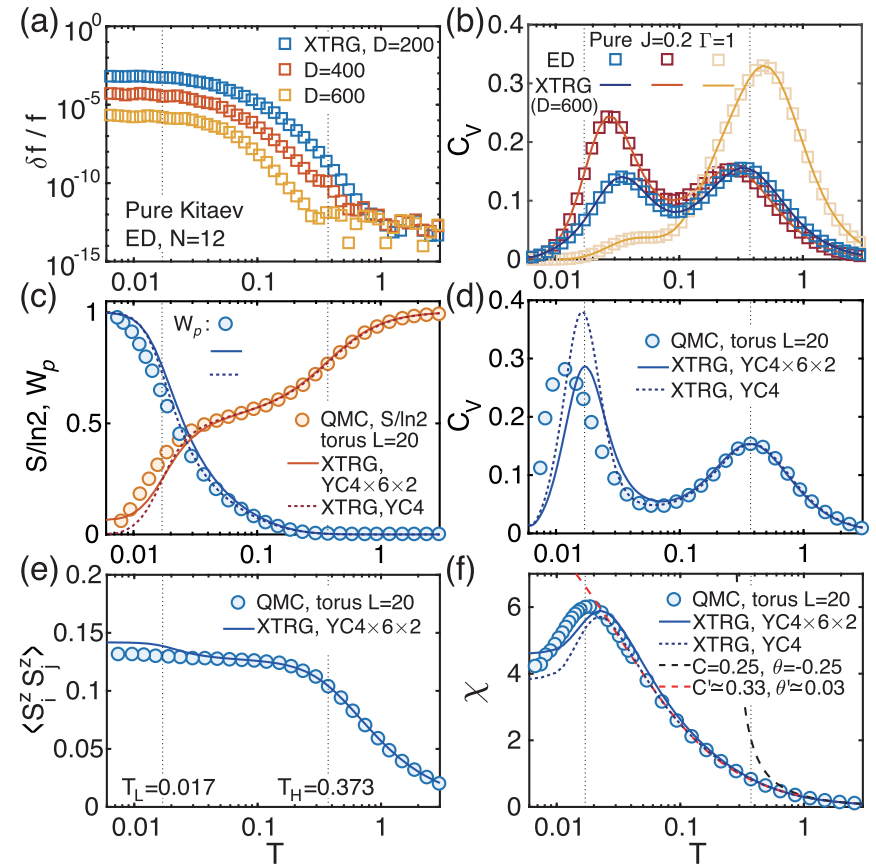

FIG. 10. (a) Relative errors $\delta f / f$ between the ED and XTRG results of a isotropic pure Kitaev model on the $N=12$ (YC4) cluster, with retained bond dimensions $D=200,400$ and 600. (b) Corresponding specific-heat results of the pure Kitaev model (blue), the Kitaev-Heisenberg model with $J=0.2$ (red), and the Kitaev- $\Gamma$ model with $\Gamma=1$ (yellow). Comparisons between the large-scale XTRG and QMC simulations [27] are presented for (c) the thermal entropy $S / \ln 2$ (orange) and flux $W_{P}$ (blue), (d) specific heat $C_{V}$, and (e) spin correlations $\left\langle S_{i}^{z} S_{j}^{z}\right\rangle$. We also compare the XTRG susceptibility results to the QMC data on the $L=20$ torus [29], where the highand intermediate- $T$ fittings are also performed on the latter (dashed lines). We show in (c)-(f) both the YC $4 \times 6 \times 2$ results (solid curves) and the YC4 data (dotted curves) obtained by subtracting length $L=6$ by $L=4$ results. The XTRG results of susceptibility are computed under the [001] field, which agree excellently with the QMC results in (f) above the low temperature $T_{L}$, although the latter is along the [111] direction.

liquid regime. The modest deviation of the XTRG results from QMC data near the lower-temperature scale $T_{L}$ can be ascribed to the cylindrical boundary condition and the finite width, which nevertheless does not lead to any qualitative difference for our discussion.

Moreover, we have also analyzed the large-scale QMC susceptibility data in Fig. 10(f), and from the fitting we find again an emergent Curie behavior clearly at intermediate temperature. The Curie constant $C^{\prime} \simeq \frac{1}{3}$ is also very close to the YC4 XTRG results in Fig. 5(a).

\section{APPENDIX B: SPIN CORRELATIONS IN THE FRACTIONAL LIQUID}

The real-space spin correlations of the extended Kitaev models are computed at a high $(T \simeq 1)$ and an intermediate temperature $(T \simeq 0.1)$. In Figs. 11(a) and 11(b) we provide $\left\langle S_{i}^{z} S_{j}^{z}\right\rangle$ along two different (zigzag and armchair) paths, under the influence of external fields, from which we find that the correlations are bond directional and extremely short
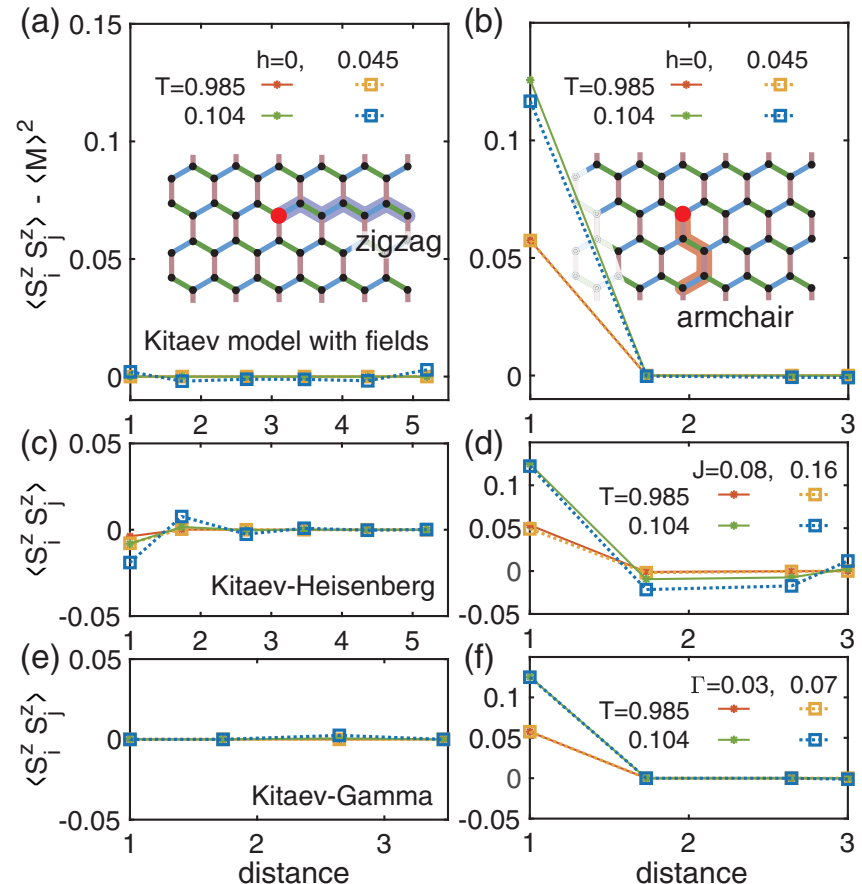

FIG. 11. High- and intermediate- $T$ spin correlations of the extended Kitaev model, along the (a), (c), and (e) zigzag and (b), (d), and (f) armchair paths, with the reference site marked in red and the hexagon edge length as the unit of distance. Note that in (a) and (b) we have subtracted the (field-induced) mean magnetization squared $\langle M\rangle^{2}$ from the spin correlations.

ranged. To be specific, $\left\langle S_{i}^{z} S_{j}^{z}\right\rangle$ vanishes along the zigzag path in Fig. 11(a), since there are only $x$ and $y$ bonds there. On the other hand, in Fig. 11(b) the armchair correlations decay instantaneously for distances exceeding one and the nearest $z$-bond correlation is enhanced as temperature decreases from $T \simeq 1$ to $T \simeq 0.1$. For the Kitaev-Heisenberg and Kitaev- $\Gamma$ models in Figs. 11(c)-11(f), we observe real-space correlation features very similar to those in Figs. 11(a) and 11(b), except for the curves with $J=0.16$ and $T \simeq 0.1$ in Fig. 11(d). There the system enters the SAF-ordered phase, where the zigzag correlations develops a small value and the signs alternate following the SAF pattern as shown in the inset of Fig. 6(a).

From the results in Fig. 11, we find spatially anisotropic and extremely-short-ranged spin correlations at intermediate temperature. This, together with the distinct stripy spinstructure factors in the main text, provide the signatures of fraction liquid.

\section{APPENDIX C: MATRIX PRODUCT OPERATOR ENTANGLEMENT}

In the MPO form of the density matrix $\rho(\beta \equiv 1 / T)$, we compute the bipartite entanglement entropy $S_{E}(\beta)=$ $-\operatorname{Tr}\left(\tilde{\rho}_{\mathrm{A}} \ln \tilde{\rho}_{\mathrm{A}}\right)$, where $\tilde{\rho}_{A}$ is the reduced matrix of subsystem $A$ (chosen as half the system) in the superstate $|\rho(\beta)\rangle$ purified from the thermal density matrix $\rho(\beta)$. In the XTRG method, $S_{E}(\beta)$ can be used to measure the required computational resource, since the bond dimension scales as $D \sim e^{S_{E}(\beta)}$ for an accurate simulation down to a temperature $T \equiv 1 / \beta$. For 
a gapped system, $S_{E}$ typically saturated as $T$ is below the excitation gap $\Delta$, and thus one can in principle simulate the low- $T$ properties even down to the ground state with a sufficiently large, but finite, bond dimension $D$. The logarithmic entanglement scaling $S_{E}(\beta) \sim \eta \ln \beta$ requires $D \propto \beta^{\eta}$, with $\eta$ an algebraic exponent. The logarithmic entanglement scaling at low temperature usually corresponds to gapless excitations. Some typical examples include the low- $T$ thermal states near the $1+1 \mathrm{D}$ conformal critical point and in the $2 \mathrm{D}$ Heisenberg model with Goldstone modes [34,36]. In the former case, the exponent has been found to be universal, $\eta=c / 3$, with $c$ the central charge of the conformal field theory [34].

In Fig. 12(a) we show the bipartite entanglement entropies $S_{E}$ across the center of the system under various magnetic fields $h$, which exhibits a logarithmic scaling as $S_{E} \sim \frac{2}{3} \ln \beta$ in the intermediate- $T$ regime, as indicated by the black dashed line. In Figs. 12(b) and 12(c) we have also computed the MPO entanglement for the Kitaev-Heisenberg and Kitaev- $\Gamma$ models, where similar logarithmic behaviors are also observed in the fractional liquid regime. As the coupling strength increases, for all three cases in Figs. 12(a)-(c), the entanglement $S_{E}$ deviates from the logarithmic behavior, exhibits a shoulder/peak, and eventually converges to a constant at sufficiently low temperature.

The exponent $\eta \approx 2 / 3$ suggests that the required computational resource for simulating the Kitaev models at intermediate temperature is similar to a conformal critical point with central charge $c=2$. In addition, in Fig. 12(a) the maximal value of $S_{E}$ appears at the lowest temperature and is less than 3.5 , which reveals the accessibility of the YC $4 \times 6 \times 2$ systems by retaining not too many bond states. In fact, we have plotted in Fig. 12(a) the $D=400$ and 600 results, where a very good convergence can be seen.

\section{APPENDIX D: MAGNETIC SUSCEPTIBILITY ALONG VARIOUS FIELD DIRECTIONS}

In Fig. 13 we show the $\chi$ data along various field directions, as well as the case $h \|[001]$ considered in the main text. We label the field direction $l \hat{h}_{x}+m \hat{h}_{y}+n \hat{h}_{z}$ by $[l, m, n]$, where $\hat{h}_{x}, \hat{h}_{y}$, and $\hat{h}_{z}$ are three cubic axes in the spin space; therefore, the Zeeman term reads

$$
H_{f}=-\sum_{i} \frac{h}{\sqrt{l^{2}+m^{2}+n^{2}}}\left(l S_{i}^{x}+m S_{i}^{y}+n S_{i}^{z}\right) .
$$

First, we apply an in-plane field $h \|[-101]$, whose direction is shown in Fig. 13(a), and compute the susceptibility. The results are shown in Figs. 13(b) and 13(c), where an intermediate Curie behavior can be clearly identified. For the out-of-plane field $h$ perpendicular to the $a b$ plane, i.e., $h \|$ [111], a Curie susceptibility also emerges [see Fig. 10(f)], as already discussed in Appendix A. As for more field directions, we perform ED calculations on systems of very limited size, $N=12$ clusters, and show the results in Fig. 13(d). A diverging susceptibility behavior near the high- $T$ scale $T_{H}$ is also evident, confirming that the emergent Curie-law susceptibility is robust and ubiquitous in the Kitaev model. This universal susceptibility scaling can be observed under various fields of different directions, on different lattice sizes/geometries,
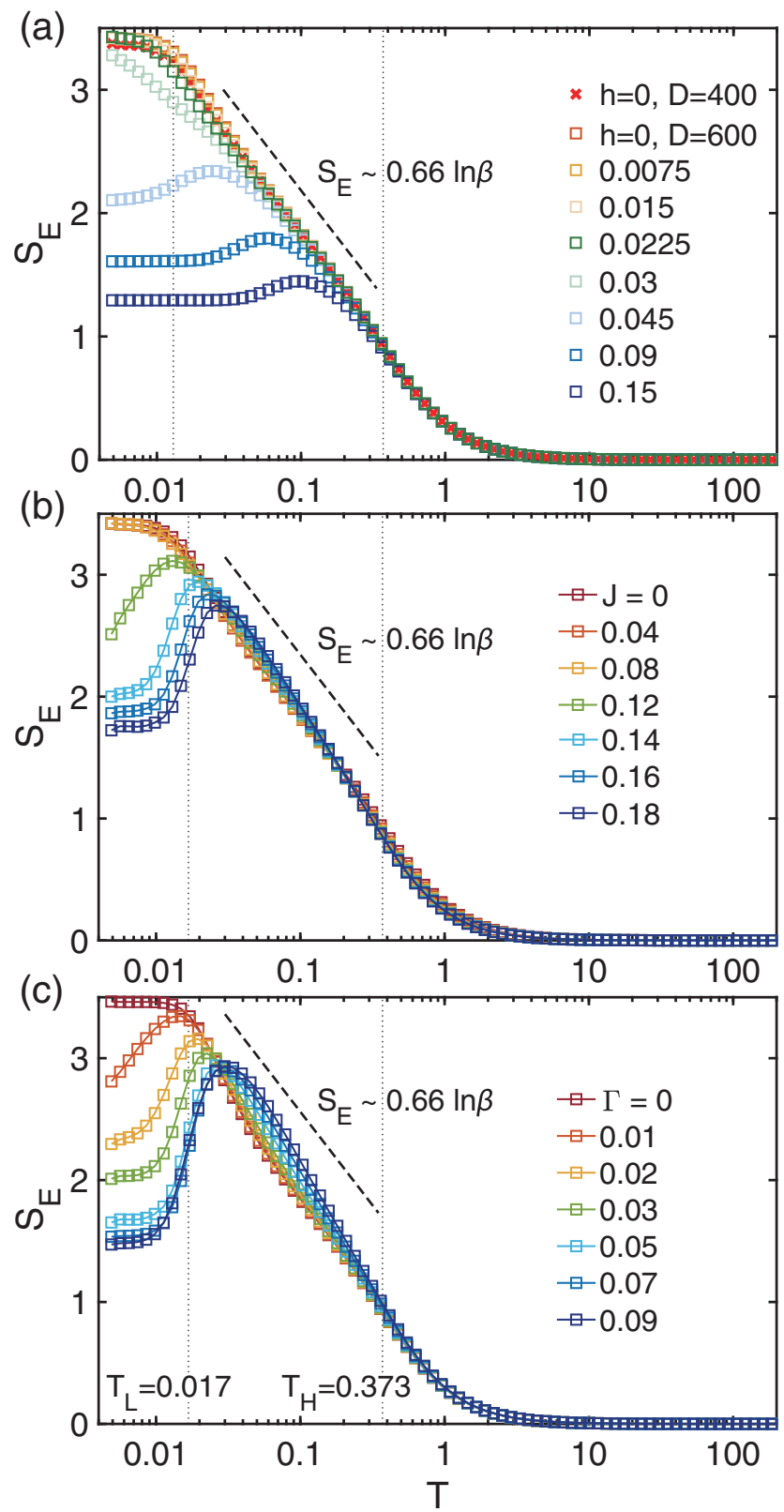

FIG. 12. Bipartite entanglement $S_{E}$ cut at the center of the MPO representation of density matrices for (a) the Kitaev model with fields, (b) the Kitaev-Heisenberg model, and (c) the Kitaev- $\Gamma$ model. At intermediate temperature, $S_{E}$ roughly scales as $\sim 2 / 3 \ln \beta$, which characterizes the required computational resource of the simulations.

and by different methods including the ED, QMC, and XTRG approaches.

\section{APPENDIX E: KUBO FORMULA OF MAGNETIC SUSCEPTIBILITY}

Here we derive the Kubo formula of the magnetic susceptibility, in terms of imaginary-time dynamical correlations. Suppose the magnetization of the system is uniform and the magnetic moment per spin, under a perturbative magnetic field $h$ along the $z$ axis, is the expectation value of $S^{z}$ on an 

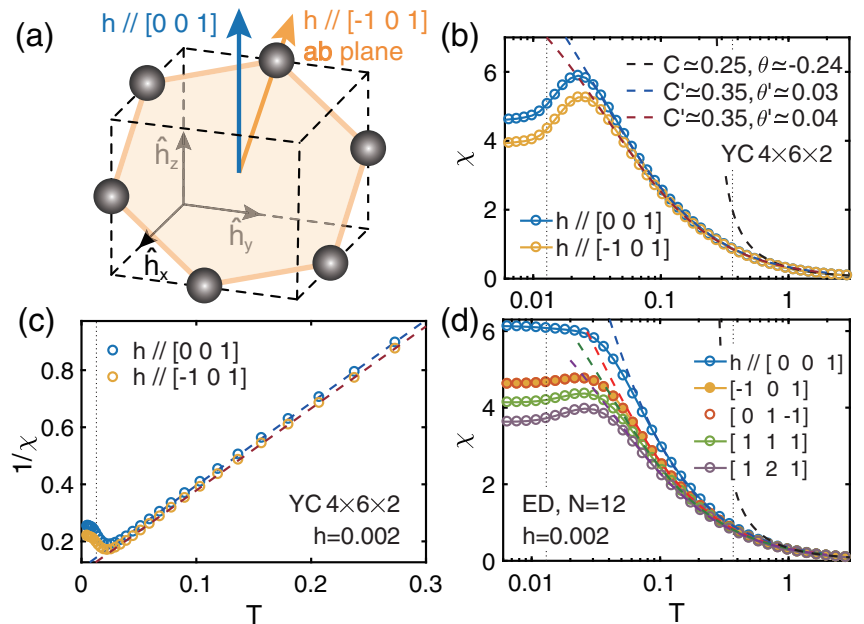

FIG. 13. (a) Illustration of the magnetic fields $h$ along [001] and [-101] (parallel to the honeycomb $a b$ plane). Also shown are (b) the magnetic susceptibility $\chi$ and (c) its inverse $1 / \chi$, calculated with a small field $h=0.002$. (d) Susceptibility results along various field directions, obtained by ED calculations on the $N=12$ cluster.

arbitrary site $i_{0}$,

$$
M=\left\langle S_{i_{0}}^{z}\right\rangle_{\beta}=\frac{1}{Z(\beta)} \operatorname{Tr}\left[S_{i_{0}}^{z} e^{-\beta H}\right],
$$

where $H(K, h)=H_{0}(K)-h S_{\text {tot }}^{z}, H_{0}(K)$ is the pure Kitaev Hamiltonian, and $S_{\mathrm{tot}}^{z}=\sum_{j} S_{j}^{z}$ is the total magnetization operator. The magnetic susceptibility $\chi=\partial M / \partial h$ can be obtained by taking the derivative of Eq. (E1). Note that since $\left[H, S_{\text {tot }}^{z}\right] \neq$ 0 , the derivative of the exponent $-\beta \partial H / \partial h=-\beta S_{\text {tot }}^{z}$ cannot be taken straightforwardly from the exponential, i.e.,

$$
\frac{\partial}{\partial h} e^{-\beta H} \neq-\beta \frac{\partial H}{\partial h} e^{-\beta H} .
$$

Instead, for an operator $O(u)$ as a function of $u$,

$$
\frac{\partial}{\partial u} e^{O}=\int_{0}^{1} e^{\lambda O} \frac{\partial O}{\partial u} e^{(1-\lambda) O} d \lambda .
$$

This can be proved via the decomposition $e^{O}=\left(e^{O / N}\right)^{N}$, and for sufficiently large $N$ it satisfies $\partial e^{O / N} / \partial u=$ $e^{O / N}(\partial O / \partial u) / N$. Therefore,

$$
\begin{aligned}
\frac{\partial}{\partial u} e^{O} & =\sum_{n=1}^{N}\left(e^{O / N}\right)^{(n-1)} \frac{\partial e^{O / N}}{\partial u}\left(e^{O / N}\right)^{(N-n)} \\
& \simeq \frac{1}{N} \sum_{n=1}^{N} e^{(n / N) O} \frac{\partial O}{\partial u} e^{(1-n / N) O} .
\end{aligned}
$$

Taking the limit $N \rightarrow \infty$, we arrive at Eq. (E3). With this, we can express the susceptibility as

$$
\begin{aligned}
\chi & =\frac{\beta}{Z(\beta)} \int_{0}^{1} \operatorname{Tr}\left[S_{i_{0}}^{z} e^{-\lambda \beta H} S_{\mathrm{tot}}^{z} e^{-(1-\lambda) \beta H}\right] d \lambda-\beta\left\langle S_{i_{0}}^{z}\right\rangle_{\beta}\left\langle S_{\mathrm{tot}}^{z}\right\rangle_{\beta} \\
& =\frac{1}{Z(\beta)} \int_{0}^{\beta} \operatorname{Tr}\left[S_{i_{0}}^{z} e^{-\tau H} S_{\mathrm{tot}}^{z} e^{(\tau-\beta) H}\right] d \tau-\beta\left\langle S_{i_{0}}^{z}\right\rangle_{\beta}\left\langle S_{\mathrm{tot}}^{z}\right\rangle_{\beta}
\end{aligned}
$$

$$
\begin{aligned}
& =\frac{1}{Z(\beta)} \int_{0}^{\beta} \operatorname{Tr}\left[e^{\tau H} S_{i_{0}}^{z} e^{-\tau H} S_{\mathrm{tot}}^{z} e^{-\beta H}\right] d \tau-\beta\left\langle S_{i_{0}}^{z}\right\rangle_{\beta}\left\langle S_{\mathrm{tot}}^{z}\right\rangle_{\beta} \\
& =\sum_{j} \int_{0}^{\beta}\left\langle S_{i_{0}}^{z}(\tau) S_{j}^{z}\right\rangle_{\beta} d \tau-\beta\left\langle S_{i_{0}}^{z}\right\rangle_{\beta}\left\langle S_{\mathrm{tot}}^{z}\right\rangle_{\beta}
\end{aligned}
$$

where in the second line we have made the variable substitution $\tau \equiv \lambda \beta$ and in the penultimate line we perform a cyclic permutation within the trace. For the sake of simplicity (and without loss of generality), we assume $M=\left\langle S_{i_{0}}^{z}\right\rangle_{\beta}=0$ in the discussion of fractional liquid, and thus arrive at the Kubo formula (3).

If the total spin $S_{\text {tot }}^{z}$ commutes with the Hamiltonian $H$, e.g. in the Heisenberg model, the two factors $e^{\tau H}$ and $e^{-\tau H}$ cancel each other and the Kubo formula reduces to the conventional susceptibility expression in terms of equal-time spin correlators. Here in the Kitaev model, although $S_{\text {tot }}^{z}$ is not a good quantum number, $e^{\tau H}$ and $e^{-\tau H}$ nevertheless approximately cancel in the fractional liquid regime (see the discussion in Sec. IV) and Eq. (5) also holds, which in turn gives rise to a Curie behavior with a modified Curie constant $C^{\prime}>\frac{1}{4}$.
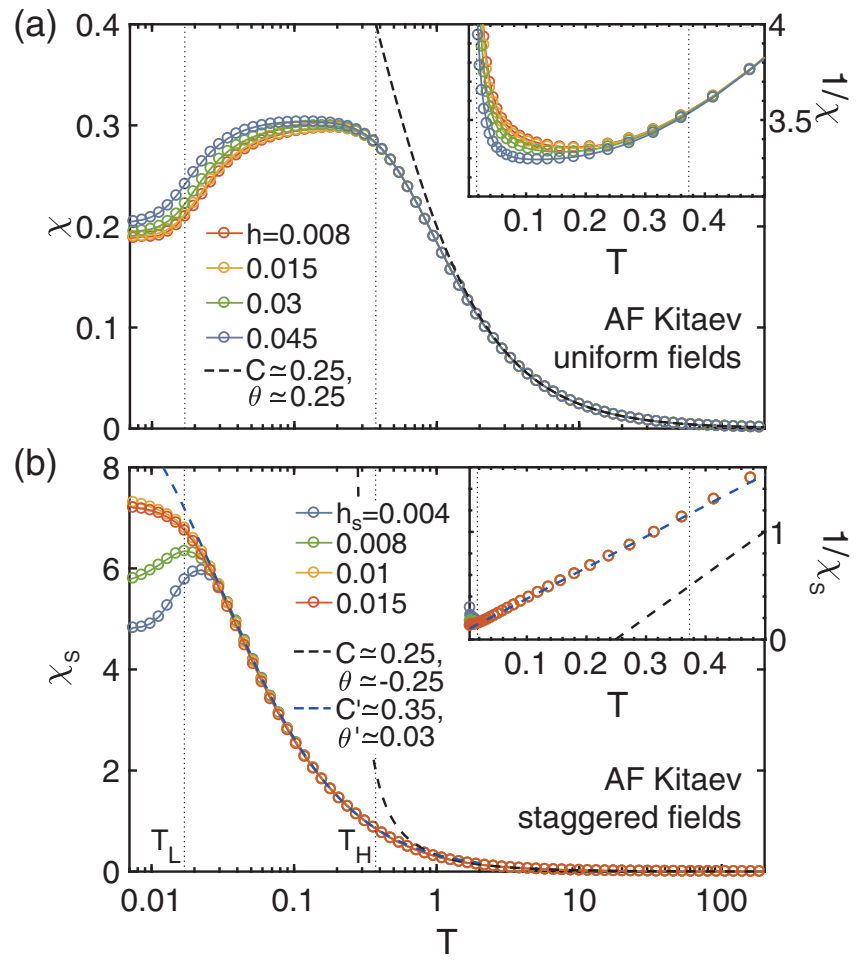

FIG. 14. (a) Uniform susceptibility $\chi$ of the AF Kitaev model under various fields $h$. The black dashed line indicates the high- $T$ Curie-Weiss fitting, and at intermediate temperature no emergent Curie behavior is found, which is also evident as shown in the $1 / \chi$ plot (inset). Since the AF and FM Kitaev models are exactly equivalent under zero fields, here the two temperature scales $T_{L}$ and $T_{H}$ coincide with those in Fig. 5(a). (b) Staggered susceptibility $\chi_{S}$ of AF Kitaev model exhibiting a universal Curie behavior in the intermediate- $T$ regime (see also $1 / \chi_{S}$ in the inset). In fact, the $\chi_{S}$ data of the AF Kitaev model are in quantitative agreement with the uniform susceptibility $\chi$ of the FM Kitaev model. 

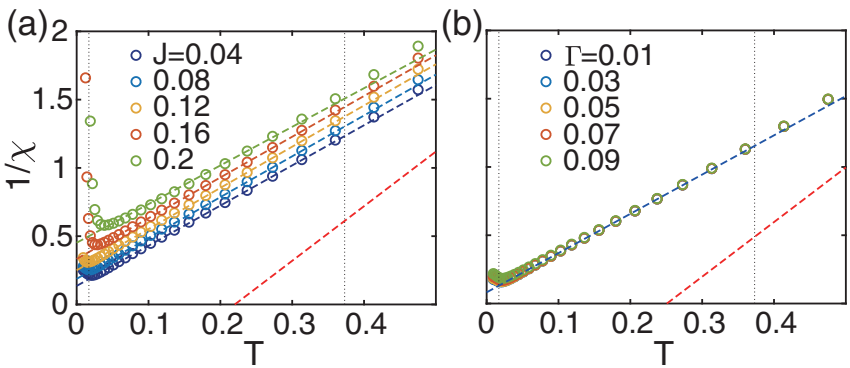

FIG. 15. Inverse susceptibility $1 / \chi$ of (a) the Kitaev-Heisenberg and (b) Kitaev- $\Gamma$ models with various $J$ or $\Gamma$ interactions. The linear fittings are shown in both the high- $T$ (the red dashed lines) and intermediate- $T$ regimes, with the same fitting parameters as those in Figs. 6(g) and 6(h).

However, as the temperature further decreases to the KSL regime, the dynamical correlation decays exponentially as the gauge fluxes freeze at low temperature below the finite flux excitation gap, which can suppress the universal Curie-law susceptibility at intermediate temperature.

\section{APPENDIX F: UNIFORM AND STAGGERED MAGNETIC SUSCEPTIBILITIES IN THE ANTIFERROMAGNETIC KITAEV MODEL}

For the isotropic Kitaev model with the AF Kitaev interaction $K_{x, y, z}=1$, we also compute the uniform magnetic susceptibility $\chi$ by applying small fields that coupled to the $S^{z}$ component, i.e., along the [001] direction. In sharp contrast to the FM Kitaev model results in Fig. 5, the uniform susceptibilities $\chi$ in Fig. 14(a) do not exhibit a universal Curie behavior in the intermediate- $T$ regime. The susceptibility curves there are nondiverging convex curves and cannot be fitted by the Curie-Weiss formula. Our cylindrical results of AF Kitaev susceptibility in Fig. 14(a) are again in excellent agreement with previous QMC results [29].

The underlying reason for the absence of emergent Curie behaviors under uniform fields is that the nearest spin pairs in the AF Kitaev model are also correlated along the $d_{z}$ bond, but in an antiparallel manner. Therefore, the change of total spin that relates to uniform susceptibility corresponds to flipping one of the two spins, which has to overcome a finite amount of AF binding energy along the $d_{z}$ bond and does not reflect the fluctuating fluxes. In fact, as shown in Fig. 14(b), the staggered susceptibility $\chi_{S}$ under a staggered magnetic field $h_{S}$ (which has alternating sign on $A$ and $B$ sublattices of the Kitaev honeycomb) exhibits a universal Curie behavior at intermediate temperature.
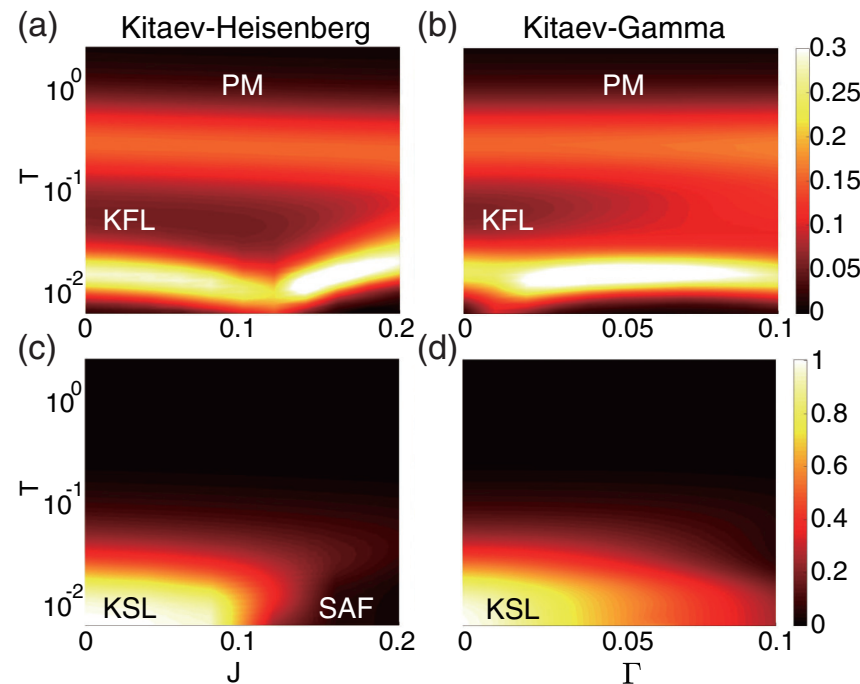

FIG. 16. Landscape of specific-heat data for the (a) KitaevHeisenberg and (b) Kitaev- $\Gamma$ models. The corresponding gauge fluxes $W_{P}$ are contour plotted in (c) and (d) for the two models.

\section{APPENDIX G: THERMODYNAMICS OF THE KITAEV-HEISENBERG AND KITAEV- $\Gamma$ MODELS}

In this Appendix we provide additional thermodynamic results of the Kitaev-Heisenberg and Kitaev- $\Gamma$ models. In Fig. 15 we plot the inverse susceptibility data $1 / \chi$ vs $T$, under the Heisenberg [Fig. 15(a)] and $\Gamma$ [Fig. 15(b)] interactions. The emergent Curie-Weiss susceptibility scaling is evident at intermediate temperature in Fig. 15, complementing the $\chi$ vs $T$ fittings in Figs. 6(g) and 6(h).

In Fig. 16 we show contour plots of the specific heat $C_{V}$ and gauge flux $W_{P}$ results of both models. From Figs. 16(a) and $6(\mathrm{~b})$ we see that the $C_{V}$ curves are always double peaked, which defines two temperature scales $T_{H}$ and $T_{L}$ that bound the fractional liquid regime. The corresponding flux expectations $W_{P}$ are plotted in Figs. 16(c) and 16(d), where the asymptotic KSL regimes with nonzero $W_{P}$ can be clearly identified.

For the Kitaev-Heisenberg model, the low- $T$ scale $T_{L}$ determined from the lower peak position of $C_{V}$ exhibits a clear $\operatorname{dip}$ at $J_{c} \simeq 0.12$ in Fig. 16(a) and for $J \lesssim J_{c} W_{P}$ quickly establishes itself at around $T_{L}$ as shown in Fig. 16(c). The scenario is altered for $J \gtrsim J_{c}$, where a different low- $T$ scale $T_{L}^{\prime}$ emerges, which represents the temperature scale between the SAF phase and Kitaev fractional liquid. In contrast, for the Kitaev- $\Gamma$ model in Fig. 16(b), the low- $T$ scale $T_{L}$ remains stable for various $\Gamma$ values, suggesting that the low- $T$ KSL regime is very robust against $\Gamma$ interactions. This is also supported by the flux results in Fig. 16(d), where $W_{P}$ remains nonzero at low temperature at least up to $\Gamma=0.1$.
[1] P. W. Anderson, Resonating valence bonds: A new kind of insulator? Mater. Res. Bull. 8, 153 (1973).

[2] L. Balents, Spin liquids in frustrated magnets, Nature (London) 464, 199 (2010).
[3] Y. Zhou, K. Kanoda, and T.-K. Ng, Quantum spin liquid states, Rev. Mod. Phys. 89, 025003 (2017).

[4] A. P. Ramirez, Geometric frustration: Magic moments, Nature (London) 421, 483 (2003). 
[5] C. Lacroix, P. Mendels, and F. Mila, Introduction to Frustrated Magnetism: Materials, Experiments, Theory (Springer, Berlin, 2011).

[6] A. Kitaev, Anyons in an exactly solved model and beyond, Ann. Phys. (NY) 321, 2 (2006).

[7] M. Hermanns, I. Kimchi, and J. Knolle, Physics of the Kitaev model: Fractionalization, dynamic correlations, and material connections, Annu. Rev. Condens. Matter Phys. 9, 17 (2018).

[8] A. Kitaev, Fault-tolerant quantum computation by anyons, Ann. Phys. (NY) 303, 2 (2003).

[9] S. Trebst, Kitaev Materials, Lecture Notes of the 48th IFF Spring School "Topological Matter-Topological Insulators, Skyrmions and Majoranas,” Jülich, 2017, arXiv:1701.07056.

[10] S. M Winter, A. A Tsirlin, M. Daghofer, J. van den Brink, Y. Singh, P. Gegenwart, and R. Valentí, Models and materials for generalized Kitaev magnetism, J. Phys.: Condens. Matter 29, 493002 (2017).

[11] H. Takagi, T. Takayama, G. Jackeli, G. Khaliullin, and S. E. Nagler, Concept and realization of Kitaev quantum spin liquids, Nat. Rev. Phys. 1, 264 (2019).

[12] G. Jackeli and G. Khaliullin, Mott Insulators in the Strong SpinOrbit Coupling Limit: From Heisenberg to a Quantum Compass and Kitaev Models, Phys. Rev. Lett. 102, 017205 (2009).

[13] J. Chaloupka, G. Jackeli, and G. Khaliullin, Kitaev-Heisenberg Model on a Honeycomb Lattice: Possible Exotic Phases in Iridium Oxides $A_{2} \mathrm{IrO}_{3}$, Phys. Rev. Lett. 105, 027204 (2010).

[14] Y. Singh and P. Gegenwart, Antiferromagnetic Mott insulating state in single crystals of the honeycomb lattice material $\mathrm{Na}_{2} \mathrm{IrO}_{3}$, Phys. Rev. B 82, 064412 (2010).

[15] Y. Singh, S. Manni, J. Reuther, T. Berlijn, R. Thomale, W. $\mathrm{Ku}, \mathrm{S}$. Trebst, and P. Gegenwart, Relevance of the HeisenbergKitaev Model for the Honeycomb Lattice Iridates $A_{2} \mathrm{IrO}_{3}$, Phys. Rev. Lett. 108, 127203 (2012).

[16] J. Chaloupka, G. Jackeli, and G. Khaliullin, Zigzag Magnetic Order in the Iridium Oxide $\mathrm{Na}_{2} \mathrm{IrO}_{3}$, Phys. Rev. Lett. 110, 097204 (2013).

[17] S. H. Chun, J.-W. Kim, J. Kim, H. Zheng, C. C. Stoumpos, C. D. Malliakas, J. F. Mitchell, K. Mehlawat, Y. Singh, Y. Choi, T. Gog, A. Al-Zein, M. M. Sala, M. Krisch, J. Chaloupka, G. Jackeli, G. Khaliullin, and B. J. Kim, Direct evidence for dominant bond-directional interactions in a honeycomb lattice iridate $\mathrm{Na}_{2} \mathrm{IrO}_{3}$, Nat. Phys. 11, 462 (2015).

[18] K. Mehlawat, A. Thamizhavel, and Y. Singh, Heat capacity evidence for proximity to the Kitaev quantum spin liquid in $A_{2} \mathrm{IrO}_{3}$ ( $\left.A=\mathrm{Na}, \mathrm{Li}\right)$, Phys. Rev. B 95, 144406 (2017).

[19] K. Kitagawa, T. Takayama, Y. Matsumoto, A. Kato, R. Takano, Y. Kishimoto, S. Bette, R. Dinnebier, G. Jackeli, and H. Takagi, A spin-orbital-entangled quantum liquid on a honeycomb lattice, Nature (London) 554, 341 (2018).

[20] K. W. Plumb, J. P. Clancy, L. J. Sandilands, V. V. Shankar, Y. F. $\mathrm{Hu}, \mathrm{K}$. S. Burch, H.-Y. Kee, and Y.-J. Kim, $\alpha-\mathrm{RuCl}_{3}$ : A spinorbit assisted Mott insulator on a honeycomb lattice, Phys. Rev. B 90, 041112(R) (2014).

[21] J. A. Sears, M. Songvilay, K. W. Plumb, J. P. Clancy, Y. Qiu, Y. Zhao, D. Parshall, and Y.-J. Kim, Magnetic order in $\alpha-\mathrm{RuCl}_{3}$ : A honeycomb-lattice quantum magnet with strong spin-orbit coupling, Phys. Rev. B 91, 144420 (2015).

[22] A. Banerjee, C. A. Bridges, J. Q. Yan, A. A. Aczel, L. Li, M. B. Stone, G. E. Granroth, M. D. Lumsden, Y. Yiu, J. Knolle,
S. Bhattacharjee, D. L. Kovrizhin, R. Moessner, D. A. Tennant, D. G. Mandrus, and S. E. Nagler, Proximate Kitaev quantum spin liquid behavior in a honeycomb magnet, Nat. Mater. 15, 733 (2016).

[23] A. Banerjee, J. Yan, J. Knolle, C. A. Bridges, M. B. Stone, M. D. Lumsden, D. G. Mandrus, D. A. Tennant, R. Moessner, and $\mathrm{S}$. E. Nagler, Neutron scattering in the proximate quantum spin liquid $\alpha-\mathrm{RuCl}_{3}$, Science 356, 1055 (2017).

[24] S.-H. Do, S.-Y. Park, J. Yoshitake, J. Nasu, Y. Motome, Y. S Kwon, D. T. Adroja, D. J. Voneshen, K. Kim, T.-H. Jang, J.-H. Park, K.-Y. Choi, and S. Ji, Majorana fermions in the Kitaev quantum spin system $\alpha-\mathrm{RuCl}_{3}$, Nat. Phys. 13, 1079 (2017).

[25] J. Nasu, M. Udagawa, and Y. Motome, Vaporization of Kitaev Spin Liquids, Phys. Rev. Lett. 113, 197205 (2014).

[26] J. Nasu, M. Udagawa, and Y. Motome, Thermal fractionalization of quantum spins in a Kitaev model: Temperature-linear specific heat and coherent transport of Majorana fermions, Phys. Rev. B 92, 115122 (2015).

[27] Y. Motome and J. Nasu, Hunting Majorana fermions in Kitaev magnets, J. Phys. Soc. Jpn. 89, 012002 (2020).

[28] J. Yoshitake, J. Nasu, and Y. Motome, Fractional Spin Fluctuations as a Precursor of Quantum Spin Liquids: Majorana Dynamical Mean-Field Study for the Kitaev Model, Phys. Rev. Lett. 117, 157203 (2016).

[29] J. Yoshitake, J. Nasu, and Y. Motome, Temperature evolution of spin dynamics in two- and three-dimensional Kitaev models: Influence of fluctuating $Z_{2}$ flux, Phys. Rev. B 96, 064433 (2017).

[30] Y. Kasahara, T. Ohnishi, Y. Mizukami, O. Tanaka, S. Ma, K. Sugii, N. Kurita, H. Tanaka, J. Nasu, Y. Motome, T. Shibauchi, and Y. Matsuda, Majorana quantization and half-integer thermal quantum Hall effect in a Kitaev spin liquid, Nature (London) 559, 227 (2018).

[31] S. M. Winter, K. Riedl, D. Kaib, R. Coldea, and R. Valentí, Probing $\alpha-\mathrm{RuCl}_{3}$ Beyond Magnetic Order: Effects of Temperature and Magnetic Field, Phys. Rev. Lett. 120, 077203 (2018).

[32] L. Janssen and M. Vojta, Heisenberg-Kitaev physics in magnetic fields, J. Phys.: Condens. Matter 31, 423002 (2019).

[33] J. Yoshitake, J. Nasu, Y. Kato, and Y. Motome, Majoranamagnon crossover by a magnetic field in the Kitaev model: Continuous-time quantum Monte Carlo study, Phys. Rev. B 101, 100408(R) (2020).

[34] B.-B. Chen, L. Chen, Z. Chen, W. Li, and A. Weichselbaum, Exponential Thermal Tensor Network Approach for Quantum Lattice Models, Phys. Rev. X 8, 031082 (2018).

[35] L. Chen, D.-W. Qu, H. Li, B.-B. Chen, S.-S. Gong, J. von Delft, A. Weichselbaum, and W. Li, Two temperature scales in the triangular lattice Heisenberg antiferromagnet, Phys. Rev. B 99, 140404(R) (2019).

[36] H. Li, B.-B. Chen, Z. Chen, J. von Delft, A. Weichselbaum, and $\mathrm{W}$. Li, Thermal tensor renormalization group simulations of square-lattice quantum spin models, Phys. Rev. B 100, 045110 (2019).

[37] H. Li, Y.-D. Liao, B.-B. Chen, X.-T. Zeng, X.-L. Sheng, Y. Qi, Z. Y. Meng, and W. Li, Kosterlitz-Thouless melting of magnetic order in the triangular quantum Ising material $\mathrm{TmMgGaO}_{4}$, Nat. Commun. 11, 1111 (2020). 
[38] S. M. Winter, Y. Li, H. O. Jeschke, and R. Valentí, Challenges in design of Kitaev materials: Magnetic interactions from competing energy scales, Phys. Rev. B 93, 214431 (2016).

[39] S. M. Winter, K. Riedl, P. A. Maksimov, A. L. Chernyshev, A. Honecker, and R. Valentí, Breakdown of magnons in a strongly spin-orbital coupled magnet, Nat. Commun. 8, 1152 (2017).

[40] J. A. Sears, L. E. Chern, S. Kim, P. J. Bereciartua, S. Francoual, Y. B. Kim, and Y.-J. Kim, Ferromagnetic Kitaev interaction and the origin of large magnetic anisotropy in $\alpha-\mathrm{RuCl}_{3}$, Nat. Phys. 16, 837 (2020).

[41] J. G. Rau, E. K.-H. Lee, and H.-Y. Kee, Generic Spin Model for the Honeycomb Iridates beyond the Kitaev Limit, Phys. Rev. Lett. 112, 077204 (2014).

[42] K. Ran, J. Wang, W. Wang, Z.-Y. Dong, X. Ren, S. Bao, S. Li, Z. Ma, Y. Gan, Y. Zhang, J. T. Park, G. Deng, S. Danilkin, S.L. Yu, J.-X. Li, and J. Wen, Spin-Wave Excitations Evidencing the Kitaev Interaction in Single Crystalline $\alpha-\mathrm{RuCl}_{3}$, Phys. Rev. Lett. 118, 107203 (2017).

[43] V. M. Katukuri, S. Nishimoto, V. Yushankhai, A. Stoyanova, H. Kandpal, S. Choi, R. Coldea, I. Rousochatzakis, L. Hozoi, and J. van den Brink, Kitaev interactions between $J=1 / 2$ moments in honeycomb $\mathrm{Na}_{2} \mathrm{IrO}_{3}$ are large and ferromagnetic: Insights from $a b$ initio quantum chemistry calculations, New J. Phys. 16, 013056 (2014).

[44] Y.-L. Dong, L. Chen, Y.-J. Liu, and W. Li, Bilayer linearized tensor renormalization group approach for thermal tensor networks, Phys. Rev. B 95, 144428 (2017).

[45] G. Baskaran, S. Mandal, and R. Shankar, Exact Results for Spin Dynamics and Fractionalization in the Kitaev Model, Phys. Rev. Lett. 98, 247201 (2007).

[46] X.-Y. Feng, G.-M. Zhang, and T. Xiang, Topological Characterization of Quantum Phase Transitions in a Spin-1/2 Model, Phys. Rev. Lett. 98, 087204 (2007).

[47] J. Zheng, K. Ran, T. Li, J. Wang, P. Wang, B. Liu, Z.-X. Liu, B. Normand, J. Wen, and W. Yu, Gapless Spin Excitations in the Field-Induced Quantum Spin Liquid Phase of $\alpha-\mathrm{RuCl}_{3}$, Phys. Rev. Lett. 119, 227208 (2017).

[48] H.-C. Jiang, Z.-C. Gu, X.-L. Qi, and S. Trebst, Possible proximity of the Mott insulating iridate $\mathrm{Na}_{2} \mathrm{IrO}_{3}$ to a topological phase: Phase diagram of the Heisenberg-Kitaev model in a magnetic field, Phys. Rev. B 83, 245104 (2011).

[49] M. Gohlke, G. Wachtel, Y. Yamaji, F. Pollmann, and Y. B. Kim, Quantum spin liquid signatures in Kitaev-like frustrated magnets, Phys. Rev. B 97, 075126 (2018).

[50] D. Gotfryd, J. Rusnačko, K. Wohlfeld, G. Jackeli, J. Chaloupka, and A. M. Oleś, Phase diagram and spin correlations of the Kitaev-Heisenberg model: Importance of quantum effects, Phys. Rev. B 95, 024426 (2017).

[51] J. Rusnačko, D. Gotfryd, and J. Chaloupka, Kitaev-like honeycomb magnets: Global phase behavior and emergent effective models, Phys. Rev. B 99, 064425 (2019).

[52] C. Hickey and S. Trebst, Emergence of a field-driven U(1) spin liquid in the Kitaev honeycomb model, Nat. Commun. 10, 530 (2019).

[53] R. Schaffer, S. Bhattacharjee, and Y. B. Kim, Quantum phase transition in Heisenberg-Kitaev model, Phys. Rev. B 86, 224417 (2012).

[54] J. Reuther, R. Thomale, and S. Trebst, Finite-temperature phase diagram of the Heisenberg-Kitaev model, Phys. Rev. B 84, 100406(R) (2011).

[55] C. C. Price and N. B. Perkins, Critical Properties of the Kitaev-Heisenberg Model, Phys. Rev. Lett. 109, 187201 (2012).

[56] J. Wang, B. Normand, and Z.-X. Liu, One Proximate Kitaev Spin Liquid in the $K-J-\Gamma$ Model on the Honeycomb Lattice, Phys. Rev. Lett. 123, 197201 (2019).

[57] Y. Kubota, H. Tanaka, T. Ono, Y. Narumi, and K. Kindo, Successive magnetic phase transitions in $\alpha-\mathrm{RuCl}_{3}$ : XY-like frustrated magnet on the honeycomb lattice, Phys. Rev. B 91, 094422 (2015).

[58] Y. Yamaji, T. Suzuki, T. Yamada, S.I. Suga, N. Kawashima, and M. Imada, Clues and criteria for designing a Kitaev spin liquid revealed by thermal and spin excitations of the honeycomb iridate $\mathrm{Na}_{2} \mathrm{IrO}_{3}$, Phys. Rev. B 93, 174425 (2016).

[59] S. Widmann, V. Tsurkan, D. A. Prishchenko, V. G. Mazurenko, A. A. Tsirlin, and A. Loidl, Thermodynamic evidence of fractionalized excitations in $\alpha-\mathrm{RuCl}_{3}$, Phys. Rev. B 99, 094415 (2019).

[60] Y. Yamaji, Y. Nomura, M. Kurita, R. Arita, and M. Imada, FirstPrinciples Study of the Honeycomb-Lattice Iridates $\mathrm{Na}_{2} \mathrm{IrO}_{3}$ in the Presence of Strong Spin-Orbit Interaction and Electron Correlations, Phys. Rev. Lett. 113, 107201 (2014).

[61] L. Janssen, E. C. Andrade, and M. Vojta, Magnetization processes of zigzag states on the honeycomb lattice: Identifying spin models for $\alpha-\mathrm{RuCl}_{3}$ and $\mathrm{Na}_{2} \mathrm{IrO}_{3}$, Phys. Rev. B 96, 064430 (2017).

[62] Z.-X. Liu and B. Normand, Dirac and Chiral Quantum Spin Liquids on the Honeycomb Lattice in a Magnetic Field, Phys. Rev. Lett. 120, 187201 (2018).

[63] B.-B. Chen, Y.-J. Liu, Z. Chen, and W. Li, Series-expansion thermal tensor network approach for quantum lattice models, Phys. Rev. B 95, 161104(R) (2017). 